\title{
4. Intensität und Absorptionsindex der Röntgenstrahlen von Platin und Kohle ${ }^{1}$; von H. Kirschbaum.
}

Inhalt: \& 1. Probleme. - I. Methoden. \& 2. Das Elektrometer. \& 3. Das Funkenmikrometer. \$ 4. Die Röntgenröhre. \&5. Photographische Methode. $\$ 6$. Ionisierungsmethode. - If. Beobachtungen. 7. Vergleich der Intensitäten der Röntgenstralılung von Platin und Kohle. \$ 8. Der Absorptionsindex für Aluminium der Pt. und CStrahlung in Abhängigkeit von der Elektrodenspannung. \& 9. Beziehung zwischen dem elektrischen Absorptionsindex und der spezifischen Schwärzungsdifferenz. $\$ 10$. Gesichtspunkte für den Vergleich der Emissionen in verschiedenen Azimuten. $\$ 11$. Vergleich der Intensitäten der $\mathrm{Pt}$ - und C-Strahlung in verschiedenen Emissionsazimuten. \$ 12. Vergleich der Absorptionsindizes in verschiedenen Emissionsazimuten. $\$ 13$. Azimut gröBter Intensität. - III. Resultate.

\section{\$ 1. Probleme.}

Die Intensität und das Durchdringungsvermögen der Röntgenstrahlen sind von Anbeginn ihrer Entdeckung als wesentlich abhängig von der Elektrodenspannung an der Röntgenröhre erkannt worden. Röntgen ${ }^{2}$ ) stellte an der Strahlungsintensität außerhalb der Entladungsröhre an einem Fluoreszenzschirme und an den Schwärzungen photographischer Platten fest, daB die Zusammensetzung des von einer Platinantikathode einer Entladungsröhre ausgesandten Strahlengemisches - d.h. Strahlen von verschiedener Absorbierbarkeit und Intensität - hauptsächlich bedingt ist durch das Entladungspotential; und zwar wies er nach, daß allgemein die Durchlässigkeit der Körper für die Röntgenstrahlen mit zunehmendem Entlad ungspotential und mit diesem die Intensität steigt. Röntgen kontrollierte die Elektrodenspannung an einer der Röhre parallel geschalteten Funkenstrecke.

Trotzdem nun nach den grundlegenden Arbeiten Röntgens so ungemein zahlreiche Arbeiten über die Röntgenstrahlen

1) Gekürzte Inauguraldissertation des Verfassers.

2) W. C. Röntgen, Wied. Ann. 64. p. 1, 12, 18. 1898. 
ausgeführt worden sind, fehlt ${ }^{1}$ ) bis hente eine systematische Untersuchung, welche für ein bestimmtes Antikathodenmaterial eine ausgedehnte Reihe genau gemessener Elektrodenspannungen mit den Absorptionsindizes der von ihnen in einer Röntgenröhre zur Emission gebrachten Strahlen verknüpfte.

Diese Lücke auszufüllen, war eine der Aufgaben der vorliegenden Untersuchung, und zwar sollte dabei der Absorptionsindex nach der bekannten Ionisierungsmethode mit dem „,photographischen" Absorptionsindex verglichen werden.

Eine andere Aufgabe bestand darin, die zuerst von J.Stark ${ }^{2}$ ) nachgewiesene Dissymmetrie in der Emission von Röntgenstrahlen an einer Kohleantikathode noch einmal nachzuprüfen und vor allem über die Lage des Azimuts größter Intensität Beobachtungsmaterial für genau gemessene Elektrodenspannungen beizubringen; ferner sollte untersucht werden, ob die gleiche Erscheinung auch bej einer Platinantikathode sich zeigt. Zwar ist unterdes eine Untersuchung von W. Fried ri $\mathrm{ch}^{3}$ ) in dieser Richtung erschienen. Da sie indes in einem wichtigen Punkte, wie weiter unten dargelegt werden wird, Zweifel übrig läßt, so erschien es angebracht, die Beobachtungen von Stark, die auch Gegenstand einer theoretischen Diskussion ${ }^{4}$ ) gewesen sind, unter Bedingungen für genaue Messungen zu wiederholen.

Gleich hier seien die Gründe angegeben, aus welchen die vorliegende Untersuchung für eine Antikathode aus Kohle und für eine aus Platin durchgeführt worden ist. - Dieses Material bot einmal ein praktisches Interesse, weil es in der normalen Röntgenröhre verwendet wird. Sodann aber wurde es zum Vergleich mit Kohle deswegen gewählt, weil sein Atomgewicht sehr viel größer als dasjenige von Kohlenstoff ist.

Nach den Untersuchungen von Barkla ${ }^{5}$ ) besitzen die chemischen Atome im Gebiete des Röntgenspektrums charakteristische Eigenfrequenzen; Barkla hat diese zuerst in der

1) Gelegentliche Beobachtungen über Elektrodenspannung und $\mathbf{A b}$ sorptionsindex: W. Seitz, Ann. d. Phys. 27. p. 301. 1908; W. R. Ham, Phys. Rev. 30. p. 96. 1910; R. Whiddington, Proc. Roy. Soc. 85. p. 99. 1911.

2) J. Stark, Phys. Ztsohr. 10. p. 902. 1909; 11. p. 107. 1910.

3) W. Friedrieh, Ann. d. Phys. 39. p.377. 1912.

4) A. Sommerfeld, Phys. Ztschr. 10. p. 969. 1909; J. Stark, $\mathrm{Ph}_{i}$ is. Ztschr. 10. p. 579. 1909.

5) Ch. G. Barkla, Jahrb. d. Rad. u. El. 5. p. 2.1908. 
sogenannten Fluoreszenzstrahlung nachgewiesen, welche von gewissen Elementen bei Absorption primärer Röntgenstrahlen emittiert wird, und hat gefunden, daß die Absorbierbarkeit dieser Eigenstrahlung von dem Atomgewicht abhängt. Während sich für $\mathrm{Pt}$ eine intensive Fluoreszenzstrahlung ergab, zeigte C keine Fluoreszenzstrahlung in dem untersuchten Röntgenstrahlgebiet. S $\operatorname{tark}^{1}$ ) hat dann anknüpfend hieran die Vermutung geäußert, daß die Eigenstrahlung auch durch den Stoß der primären Kathodenstrahlen auf die Atome in der Antikathode zur Emission gebracht werden könne, daß diese also neben der Wiechert-Stokesschen Strahlung von seiten der gebremsten primären Kathodenstrahlen noch die linienhafte Eigenstrahlung ihrer Atome emittiere.

\section{Yethoden.}

\section{§ 2. Elelktrometer.}

Von Anfang an war in der vorliegenden Arbeit das Bestreben darauf gerichtet, für die Messung der Elektrodenspannung an der Röntgenröhre eine möglichst einwandfreie Methode anzuwenden; eine solche wurde in der Verwendung einer Influenzmaschine und eines Hochspannungselektrometers erblickt, welches im Prinzip demjenigen von Wulf ${ }^{2}$ ) nachgebildet ist.

Durch eine einfache Vorrichtung konnte ein Elektrometer für niedrige Spannung zur Messung hoher Spannungen benutzbar gemacht werden.

Ein Teil der von einer hochgeladenen Platte ausgehenden Kraftlinien wird abgeschirmt, der nicht abgeschirmte mündet in einem Tischchen, das auf den inneren isolierten Teil des ungeladenen Elektrometers gesetzt ist. Infolge der Influenz tritt Ladung in dem beweglichen inneren Teile des Instrumentes auf, welche die Blättchen bzw. Fäden zur Divergenz bringt. Der Ausschlag dieser hängt bei Ausschaltung aller anderen Einflüsse nur von dem jeweiligen Potential der hochgeladenen Platte ab.

Das für die vorliegende Arbeit benutzte Hochspannungselektrometer war, wie folgt, zusammengesetzt:

Von einem runden Grundbrett $|A|$ (vgl. nachstehende Fig. 1) erboben sich drei Holzstützen in einem gegenseitigen

1) J. Stark, Phys. Ztschr. 10. p. 579 u. 902.1909.

2) Th. Wulf, Phys. Ztschr. 11. p. 1090.1910. 
Abstand von $20 \mathrm{~cm}$, welche ihrerseits eine dem Grundbrett gleiche Holzplatte $|B|$ trugen.

Auf dem unteren Brett stand ein Elektrometer von Elster \& Geitel ${ }^{\mathbf{1}}$ ), dessen Stellschrauben zur Verhinderung einer Verschiebung in kleine Löcher in Grundbrett eingesenkt waren. Auf den Zuführungsstift $|C|$ zu dem inneren Teil des Elektrometers war ein kreisrundes Tischchen $|T|$ ans Zink-

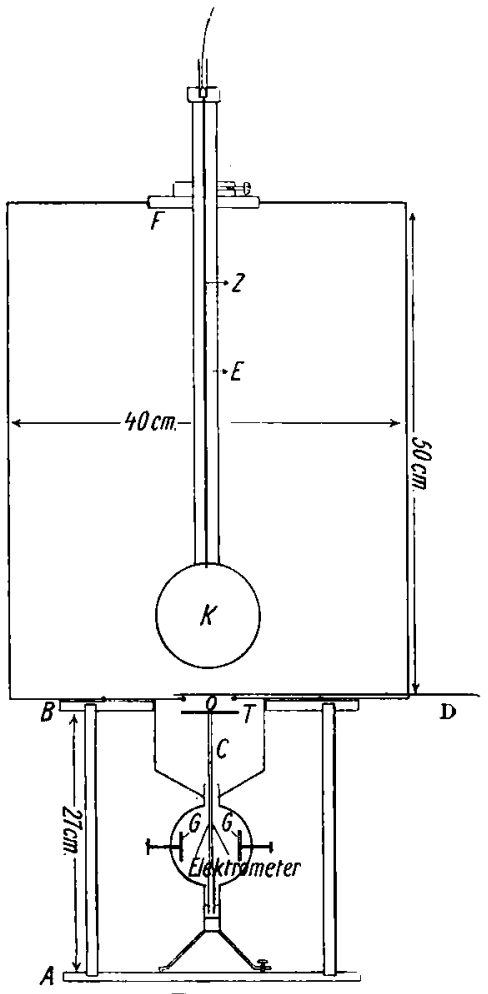

Fig. 1. blech vom Durchmesser $5,4 \mathrm{~cm}$ aufgesetzt. Die obere Holzplatte besaß eine konzentrische Öffnung von $10,5 \mathrm{~cm}$ Durchmesser, durch die ein Zinkblechzylinder nach unten hinausragte und das Tischchen und, weiterhin trichterfömig zulaufend, den oberen Teil des Elektrometergehäuses umschlo $B$.

Auf dem Holzgestell, zugleich auf einem an den Zinkblechzylinder angelöteten schmalen Band, ruhte ein runder Kasten aus Zinkblech, in dessen Boden zwei kleine Erhöhungen der oberen Holzplatte zur Verhütung einer Verschiebung eingriffen.

Der Boden des Kastens wies in der Mitte eine kreisförmige Öffnung auf, deren Rand von einem wulstigen Messingring eingefaßt war, und die durch eine Schieberklappe $|D|$ von außen her verschlossen werden konnte. Die Öffnung $|O|$ war kleiner im Durchmesser $4,8 \mathrm{~cm}$ als das Tischchen $(5,4 \mathrm{~cm})$ gewählt. Eine hohle Messingkugel $|K|$ von $10 \mathrm{~cm}$ Durchmesser wurde von einem dicken Draht $|Z|$ für die Zuleitung der Hochspannung getragen. Der Draht war zu seiner Isolierung von dem stets geerdeten Kasten in einem dickwan-

1) J. Elster u. H. Geitel, Phys. Ztschr. 4. p. 137, 1902. 
digen Hartgummirohr $|E|$ eingebettet, welches mitsamt dem Zuleitungsdraht in einer an den abnehmbaren Kastendeckel angesetzten dicken Hartgummiplatte $|F|$ verschoben und durch eine Schraube aus gleichem Material befestigt werden konnte. Auf dem Hartgummirohr war eine Millimeterskala eingeritzt.

Die Empfindlichkeit des Elektrometers warde nicht wie beim Wulfschen Hochspannungselektrometer durch Vergrößerung der Öffnung $|O|$ und die Höhe des Tischchens $|T|$ verändert, sondern nur durch den Abstand der verschiebbaren Kugel $|K|$. Es wäre nämlich bei der Größe unseres Elektrometersystems unbequem gewesen, jedesmal zur Veränderung der Empfindlichkeit für die verschiedenen hohen Spannungen das Instrument auseinanderzunehmen. Jene Anordnung empfahl sich auch schon deshalb, weil durch die Verschiebung der Kugel ein weitausgedehnter Empfindlichkeitsbereich gegeben war und - wie es sich bei den hohen Spannungen von etwa 50000 Volt zeigte - bei nicht angemessener Entfernung der Kugel vom Tischchen eine starke Aufladung des Tischchens des Elektrometers vorkam, welche stets einen heftigen Ausschlag der Blättchen bis zur Berührung der Elektrometerbacken $|G|$ nach sich zog.

Durch vielfache Beobachtungen und Messungen an dem vorbeschriebenen Elektrometer wurde festgestellt, daß es zur Bestimmung der Elektrodenspannung an der Röntgenröhre und ihres Verlaufes wohl geeignet war. - Störende Einflüsse von außen auf den Kraftlinienverlauf waren durch die allseits geschlossene Form von vornherein nicht zu befürchten. Die Empfindlichkeit konnte, wie schon erwähnt, in einem großen Bereiche verändert werden und wurde durch Verschiebung der Kugel $|K|$ meist so reduziert, daß sie 1 Proz, des Potentialwertes an der Kugel pro $1 / 10$ Skalenteil ausmachte, der noch bequem an der Elektrometerskala abgelesen werden konnte.

Die Empfindlichkeit dieses Hochspannungselektrometers konnte noch durch die Verschiebung der Backen $|G|$, die sich in dem Elektrometer von Elster \& Geitel ${ }^{\mathbf{1}}$ ) befanden, verändert werden.

Weiterhin ergab sich bei ungeänderter Kugelstellung Proportionalität zwischen dem Ausschlag am Elektrometer

1) J. Elster u. H. Geitel, Phys. Ztschr. 4, p. 137. 1902. 
und dem Potential der Kugel, wenigstens in dem mittleren Skalenbereich; für die ersten 5 Skalenteile bestand eine geringere, für die Skalenteile über 12 eine größere Empfindlichkeit des Elektrometers (vgl. hierzu Beschreibung des Elektrometers von Elster \& Geitel). ${ }^{\mathbf{}}$ )

Das Auseinandernehmen des ganzen Elektrometers, die Verschiebung der Kugel und das Wiedereinsetzen des ganzen Systems - eine Manipulation bei den Vorversuchen, die dartun sollte, ob wesentliche Veränderungen der Beziehung zwischen Ausschlag und Spannung hierdurch hervorgebracht würden ergab zuweilen eine Änderung jener Beziehung, doch überschritt die Verschiedenheit der Werte selten 1 Proz. Hin und wieder kamen Aufladungen des Elektrometers vor, die zum Teil von Staubteilchen, zum Teil von der Ionisierung der Luft zwischen Kugel und Tischchen herrühren dürften. Zur Abstellung dieser Störung genügte oft schon ein SchlieBen der Öffnung $|O|$ durch die Schieberklappe $|D|$ und Ableiten der Elektrizität durch die Flügel des Elektrometers, andererseits half das einfache Mittel des Auseinandernehmens, Reinigens und vorsichtigen Ineinandersetzens der ganzen Elektrometeranordnung.

\section{\& 3. Die Funkenstrecke.}

Die Eichung des beschriebenen Hochspannungselektrometers geschah durch eine besonders für diesen Zweck angefertigte, parallel zum Elektrometer gelegte Funkenstrecke (vgl. Fig. 2 auf der folgenden Seite).

Als Elektroden zu der Funkenstrecke wurden Messingkugeln von $5 \mathrm{~cm}$ Durchmesser genommen. Die Spannungswerte zu den einzelnen Funkenlängen wurden aus der Kurve von $M$ üller ${ }^{2}$ ) für Kugeln von $5 \mathrm{~cm}$ Durchmesser interpoliert.

Die Funkenstrecke war symmetrisch angeordnet. Die Funkenbahn lag vertikal. Die untere Elektrode $|A|$ war durch einen Glasstab $|B|$ von der Grundplatte isoliert und fest; sie erhielt die Spannung.

Die Einstellung des Elektrodenabstandes erfolgte durch Verschiebung der stets geerdeten Kugel $|C|$, deren Messing-

1) J. Elster u. H. Geitel, Phys. Zeitschr. 4. p. 137. 1902.

2) C. Müller, Ann. d. Phys. 28. p. 585. 1909. 
gestänge $|D E|$ an den Schlitten eines bis zu $6 \mathrm{~cm}$ verschiebbaren Mikrometers angesetzt war. Um auch größere Entfernungen der Kugelelektroden zu ermöglichen, konnte die die obere Kugel $|C|$ tragende senkrechte Stange $|D|$ in einer durch eine Schraube zusammendrückbare Metallhülse der Stange $|E|$ verschoben und befestigt werden.

Für alle hier in Betracht kommenden Funkenlängen reichte das Mikrometer aus.

Die Funkenstrecke war von einem Gehäuse aus Zinkblech umgeben, welches neben der oberen Öffnung für die Stange $|D|$ noch eine größere durch eine Zinkplatte verschließbare Öffnung $|O|$ aufwies; seitlich war an dem Kasten eine Hartgummiplatte eingesetzt, durch deren Mitte die Zuleitung zur unteren Elektrode ging.

Das Ganze war möglichst stabil gebaut, damit keine fehlerhafte Änderung der Elektrodenlage (Nullage usw.) während der Messungen vorkamen; so war die Grundplatte aus massivem Eisen, die Stütze, an welche das kräftige Gestänge angriff und das Mikrometer angeschraubt war, bestand aus einem starken $\mathbf{T}$ Eisen. Es wurden auch selten unliebsame Änderungen der Nullage angetroffen. Die Nullstellung der

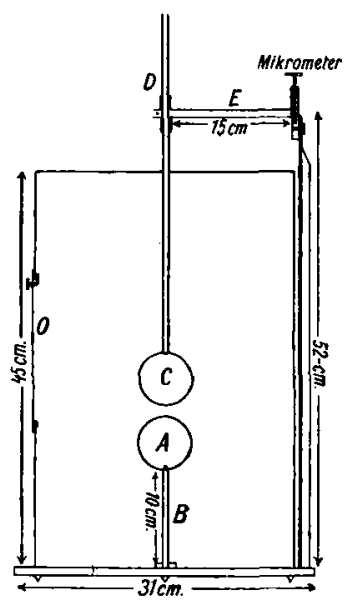

Fig. 2. Kugeln wurde dann stets durch eine Verschiebung der Vertikalstange $|D|$ wiedergegeben und vor jeder Meßreihe geprüft.

Außer der isolierten Kugelelektrode $|A|$ war das ganze Funkenmikrometer metallisch in sich verbunden und geerdet.

Die Regulierung der Spannung bis zur beabsichtigten Funkenlänge wurde durch einen parallel zu Elektrometer und Funkenstrecke geschalteten Spitzenregulator besorgt. Er war aus einem gewöhnlichen Funkenmikrometer hergestellt; statt der Kugeln stand eine mit Spitzen versehene Platte (stets negativ elektrisch) einer einfachen Platte (verschiebbar. und stets geerdet) gegenüber. 
Die Regulierung der Spannung mit den Spitzen ging ohne Schwierigkeiten.

Bei Überspringen des Funkens zwischen den Kugeln wurde der jedesmalige Ausschlag am Elektrometer für die Eichung beobachtet. - Bei ungeänderter Stellung der Kugel im Hochspannungselektrometer wiesen Ausschlag desselben und Funkenpotentialwerte keine Unregelmäßigkeiten auf.

Eine Verzögerung der Funkenentladung war durch ein im Blechgehäuse der Funkenstrecke befindliches Radiumpräparat vermieden. - Die Zuleitungsdrähte von der Influenzmaschine zu den einzelnen Instrumenten waren zur Verhütung starker Ausstrahlung der Elektrizität größtenteils in weite, mit Paraffin ausgegossene Glasröhren gebettet. An Stellen jedoch, wo eine Umwechselung oder Verschiebung des Zuleitungsdrahtes zuweilen erfolgen mußte - so an der Influenzmaschine und Röntgenröhre, am Elektrometer und Spitzenregulator -, war cler Kupferdraht von dickwandigem Weichgummi, der noch in clas Paraffin der Glasröhren hineinragte, umhüllt.

Es mag hier Erwähnung finden, daß neben den schon genannten Teilen des Elektrometers, der Funkenstrecke und des Spitzenregulators noch der positive Pol der Influenzmaschine, sowie die Antikathode der Röntgenröhre durch eine gut verlötete metallische Verbindung mit der Wasserleitung geerdet war.

\section{\$ 4. Röntgenröhre.}

Röhrenform. Zum Zweck der Verarbeitung zu einer Röntgenröhre war eine kugelförmige Röhre von $6,1 \mathrm{~cm}$ Durchmesser ausgesucht worden, welche in der Äquatorialebene senkrecht zu dem Ansatzrohr streng kreisrund war. Da in einer Äquatorialebene durch das Ansatzrohr die Dicke der Glaswand im allgemeinen besonders stark variiert, so wurde das Ansatzrohr nicht zum Kathodenrohr gemacht, sondern zur Aufnahme des Schliffstückes bestimmt, welches die Antikathode halten sollte. Das Kathodenrohr hingegen wurde in der Ebene, in welcher die Röntgenstrahlen beobachtet werden sollten - Äquatorialebene --, senkrecht zu dem Ansatzrohr angebracht.

Nach Beendigung der ganzen vorliegenden Arbeit wurde ein $3 \mathrm{~cm}$ breiter Streifen aus der benutzten Röntgenröhre so 
herausgeschnitten, daß seine Mittellinie in der Äquatorialebene senkrecht zum Ansatzrohr stand. Auf dieser Mittellinie, sowie $1 \mathrm{~mm}$ ober- und unterhalb derselben wurde mit einem Sphärometer die Glaswanddicke von $0,5 \mathrm{zu} 0,5 \mathrm{~cm}$ für verschiedene Azimute ausgemessen, und das Mittel aùs den drei Messungen genommen. Es ergaben sich die folgendem, in der Tab. 1 mitgeteilten Werte.

Tabelle 1.

\begin{tabular}{c|c|c|c}
\hline $\begin{array}{c}\text { Azimut } \\
\text { in Grad }\end{array}$ & $\begin{array}{c}\text { Glaswanddicken } \\
\text { in min }\end{array}$ & $\begin{array}{c}\text { Azimut } \\
\text { in Grad }\end{array}$ & $\begin{array}{c}\text { Glas wanddicken } \\
\text { in mm }\end{array}$ \\
\hline \hline 0 & 0,589 & 77,6 & 0,436 \\
4,5 & 0,579 & 82,2 & 0,430 \\
8,9 & 0,568 & 86,8 & 0,417 \\
13,3 & 0,555 & 91,5 & 0,411 \\
17,8 & 0,544 & 96,2 & 0,410 \\
22,3 & 0,539 & 101,0 & 0,412 \\
26,8 & 0,527 & 105,7 & 0,416 \\
31,4 & 0,528 & 110,4 & 0,422 \\
36,0 & 0,532 & 115,2 & 0,423 \\
40,6 & 0,537 & 120,0 & 0,429 \\
45,2 & 0,531 & 124,8 & 0,439 \\
49,8 & 0,526 & 129,8 & 0,451 \\
54,4 & 0,499 & 134,7 & 0,458 \\
59,0 & 0,485 & 139,6 & 0,467 \\
63,6 & 0,467 & 144,5 & 0,476 \\
68,2 & 0,455 & 149,4 & 0,488 \\
72,9 & 0,447 & - & -
\end{tabular}

Antikathode. Als Antikathodenmaterial wurde, wie schon eingangs erwähnt, Platin und Kohle verwendet.

Die Platinantikathode (vgl. Fig. 3) bestand aus einem kreisförmigen, $0,1 \mathrm{~mm}$ dicken Platinblech von $2,2 \mathrm{~cm}$ Durchmesser, welches aufliegend auf einem $2,5 \mathrm{~mm}$ breiten, $0,5 \mathrm{~mm}$ dicken Stahlring von $2,05 \mathrm{~cm}$ Durchmosser, an diesen mit Platindrähtchen befestigt war. In den Stahlring war eine in ein Gewinde eines Messingstiftes passende Spindel geschraubt. Der Messingstift war unten von einem an das Schliffstück angeschmolzenen Glasröhrchen eng umschlossen und ging selbst oben in einen Platindraht über. 
An Stelle einer Antikathode aus Buchenholzkohle wurde eine solche aus Bogenlampenkohle genommen. Dieses Material konnte bis zur Weißglut, ohne durchzubrennen, erhitzt werden. Wohl kam ein Zerspringen dieser Kohlenart vor. Es sind für die maßgebenden Beobachtungen zwei Scheiben dieser Kohle benutzt worden, welche aus dem gleichen Stück



Fig. 3. Bogenlampenkohle und in derselben Dicke geschnitten worden waren.

Die Kohlescheibe (vgl. Fig. 3 B) von $0,58 \mathrm{~mm}$ Dicke und $2,1 \mathrm{~cm}$ Durchmesser lag auf einem $0,75 \mathrm{~mm}$ dicken Platinring von $1,95 \mathrm{~cm}$ Durchmesser auf, von welchem drei angelötete Häkchen aus Platindraht auf die Vorderseite der Kohlescheibe ein wenig übergriffen, diese so haltend. Ein stärkerer Platindraht, an den Platinring mit Kupfer festgelötet, war zu einer Schraubenspindel verarbeitet, welche ebenso wie die der Platinantikathode in den Messingstift des Schliffstückes paßte.

Die Dicke der ganzen Kohleantikathode von der Spindel bis zur Kohleoberfläche $(1,3 \mathrm{~mm})$ stimmte mit der Breite von

Spindel der Platinantikathode und Platinoberfläche überein, damit sich eine möglichst genaue gleiche Azimutstellung der Antikathodenoberfläche erreichen lieB und die Emissionszentren der aus beiden Antikathoden emittierten Röntgenstrahlen die gleichen waren. Die Antikathoden ragten in die Röhrenkugel soweit hinein, daß die Auftreffstelle der Kathodenstrahlen möglichst genau in ihrer Mitte lag.

Aufstellung der Röntgenröhre. Die Röntgenröhre (vgl. Tig. 4 u. 5, p.95 u. 96) wurde unte: Zwischenschaltung eines Hahnes vermittels einer etwa $30 \mathrm{~cm}$ langen Rohrleitung an 
den Aufsatz einer Gaedepumpe, deren Vorvakuum eine rotierende Ölpumpe bediente, mit Siegellack angekittet. Das Kathodenrohr hatte horizontale, der Antikathodenträger vertikale Lage.

Der untere Teil der Glaskugel ruhte auf der Holzplatte eines stabilen Holzschemels, und, um eine Veränderung der Röhrenlage zu vermeiden, war der aufliegende Röhrenteil mit Paraffin festgegossen. Der Rötgenröhre wurde so ein genügend fester Halt geboten. Diese Befestigung der Röhre ersetzte jegliche Art von Haltern, deren Sekundärstrahlen die Beobachtung fehlerhaft hätten beeinflussen können.

Die zur Gaedepumpe führende Rohrleitung war zu Anfang seitlich an das Kathodenrohr angesetzt. Im weiteren Verlauf der Beobachtungen und Erfahrungen an der Röntgenröhre wurde diese Rohrleitung an den Antikathodenträger verlegt, um störende Entladungen nach der Pumpe zu vermeiden.

\section{§ 5. Photographische Methode.}

a) Prinzip. Das Prinzip der hier angewandten photographischen Methode nach dem Verfahren von Stark war folgendes. Es wurde um die Röntgenröhre in einigem Abstand von ihr und konzentrisch mit ihr ein photographischer Film gelegt. In dem mittleren Drittel seiner Höhe war dieser nur

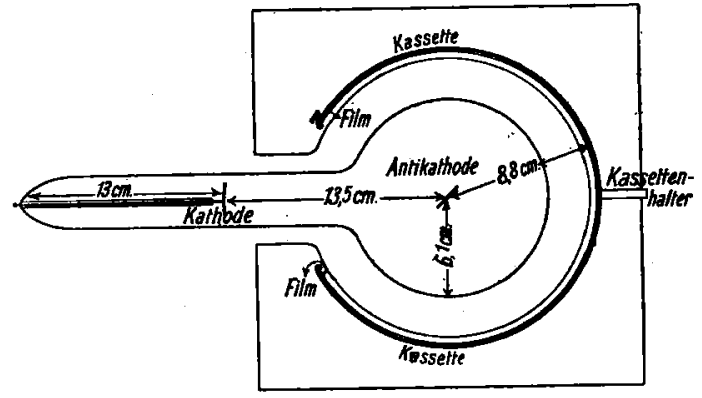

Fig. 4.

von schwarzem Papier, in seinen zwei anderen Dritteln außerdem von' Aluminiumblech bedeckt. Der Vergleich der von den Röntgenstrahlen veranlaßBten Schwärzung in seinem mittleren Drittel mit derjenigen unter dem absorbierenden AlBleche ergab für verschiedene Azimute den ,photographischen“ 
Absorptionsindex. Außerdem gestattete der Vergleich der Schwärzungen in den verschiedenen Azimuten die Feststellung der Abhängigkeit der Intensität der Röntgenstrahlung vom Azimut.

b) Kassette. Die verwendete Kassette (vgl. Fig. 4 u. 5) für den Film war streng kreisförmig. Sie war aus Messingblech hergestellt.



Fig. 5.

Indes war die zur Aufnahme des Films dienende kreis. zylindrische Wand mit einem $0,4 \mathrm{~mm}$ dicken Al-Blech bedeckt. Dieses sollte den Film vor den aus der Messingkassette rückwärts geworfenen Sekundärstrahlen schützen. Es mußte nämlich auf eine etwaige intensive Eigenstrahlung aus der Kassette unter dem Einfluß der von dieser absorbierten primären Strahlung Rücksicht genommen werden. Diese Eigenstrahlung ist nach Barklar) bei $\mathrm{Al}$ für gewöhnliche Röntgenstrahlen nicht vorhanden, wohl aber tritt sie unter Umständen bei $\mathrm{Zn}$ und $\mathrm{Cu}$ auf, also an Messing.

Der Radius der Krümmung der Kassette betrug 8,83 cm; da der Film selbst 0,2 mm, ein zwischen ihm und der Kassettenwand liegendes Papierblatt $0,1 \mathrm{~mm}$ dick war, so betrug der Radius der Krümmung der photographischen Schicht $8,8 \mathrm{~cm}$.

Damit bei allen photographischen Beobachtungen der Kassette ein fester Halt geboten wurde und ihre Stellung um

1) Ch. G. Barkla, l. c. 
die Röntgenröhre in bezug auf die Äquatorialebene gewahrt blieb, wurde folgende Einrichtung getroffen.

Um die Entladungsröhre wurde ein dickwandiger, eine weite Lücke für die Durchführung des Kathodenrohres aufweisender Holzzylinder gelegt und auf diesen eine Holzplatte fest aufgeschraubt. In die Holzplatte war eine $3 \mathrm{~mm}$ breite kreisförmige Rinne vom Radius der Kassette so tief eingeschnitten, daß der untere Rand der Kassette bis zur Metalleiste hineingesteckt werden konnte (vgl. Fig. 5).

Die Höhe des Holzzylinders und der Platte war so bemessen, dab nach Einstellung der Kassette in die Rinne die Mittellinie des photographischen Films in die Äquatorialebene der Glaskugel zu liegen kam, in der auch später die Ausmessung der Glasdicke erfolgte. Außerdem wurde vor der Befestigung dieser Einrichtung auf dem Holzsehemel mit Paraffin an der Außenseite auf iiberall gleiche Entfernung der Kassette von der Glaskugel geachtet, d. h. sie wurde konzentrisch zur Röhregestellt.

Eine Marke auf Kassette und Holzplatte ermöglichte es noch, die Enden der Kassette stets gleichweit von dem Kathodenrohr zu bringen, überhaupt übereinstimmende Stellungen derselben bei allen Beobachtungen zu erzielen.

c) Behandlung der Films. Die verwandten Films waren Agfa-Planfilms von 3,9-4 cm Breite und etwa $48 \mathrm{~cm}$ Länge. Der Filmwar stets in schwarzes, lichtdichtes Papier eingeschlagen. Auf der Vorderseite war das mittlere $7-8 \mathrm{~mm}$ breite Drittel des Films nur von diesem Papier, das obere und untere Drittel waren mit $\mathrm{Al}$-Blech von überall gleicher Dicke bedeckt, und zwar variierte bei den verschiedenen Aufnahmen je nach dem Durchdringungsvermögen der untersuchten Strahlen die Dicke des Absorptionsbleches von $0,1-0,815 \mathrm{~mm}$. In den später aufgeführten Tabellen und Kurven ist die benutzte Al-Dicke stets angegeben.

Die Entwickelung der Films geschah unter gleichen Bedingungen. $\mathrm{J}_{\theta}$ zwei Films wurden kurz gewässert und zusammen in eine große Schale, den Entwickler enthaltend, gebracht und 4 Minuten lang unter stetigem Hin- und Herbewegen der Entwickelungsschale behandelt. Die Temperatur des Entwicklers - Rodinal in einer Verdünnung von 1:15 mit Bromkalizusatz - schwankte zwischen 15-160. Nach der Entwickelung wurden die Films wiederum kurz gewässert 
und dem Fixierbad übergeben. Waren die Films gut ausfixiert, meist nach 15 Minuten, so wurden sie 1 Stunde lang fließendem Wasser ausgesetzt.

d) Messung der Schwärzungen. Die Schwärzungen der zwischen planparallelen Glasplatten gedrückten Photogramme wurden mit Hilfe des Hartmannschen Mikrophotometers meist von $1 \mathrm{~cm} \mathrm{zu} 1 \mathrm{~cm}$ ermittelt, und zwar wurde entlang den beiden äußeren Schwärzungsstreifen, welche von durch Al-Blech gegangenen Strahlung herrührten, in $2 \mathrm{~mm}$ Abstand von den Grenzen gegen die mittleren Schwärzungsstreifen und entsprechend im mittleren Schwärzungsstreifen $2 \mathrm{~mm}$ von der oberen und unteren Grenze entfernt photometriert. Aus den Schwärzungswerten (je 2) der äußeren Streifen und den zwei des mittleren Streifens wurde das Mittel genommen. Jeder einzelne Schwärzungswert wurde aus mehreren Beobachtungen gewonnen.

Es wurden auch in der Mittellinie der Schwärzung des unbedeckt gewesenen Filmteiles Photometrierungen vorgenommen, um festzustellen, ob etwa die von dem inneren Rande der Al-Bleche kommenden Sekundärstrahlen in ihrer unmittelbaren Nähe die Schwärzung fehlerhaft beeinflußt hätten, in gleicher Weise in der Mittellinie der anderen Schwärzungsstreifen. Doch wurde an mehreren Photogrammen eine solche fehlerhafte Randschwärzung nicht vorgefunden bzw. Variation der Schwärzung des bedeckt gewesenen Films nach dem Rande zu.

e) Relative Lage vom Film, Kathodenstrahlen und Emissionszentrum. Große Sorgfalt war auf die Ermittelung der Lage des Emissionszentrums der Röntgenstrahlen relativ zum Zentrum der Filmkrümmung zu legen, ferner auf die Zuordnung der einzelnen Stellen des Films zu den Emissions-Azimuten der Röntgenstrahlen. Es wurde zu diesem Zwecke folgendes Verfahren eingeschlagen.

Nach Beendigung der photographischen Aufnahmen wurde die Antikathode mit einem Fernrohr kleiner Brennweite anvisiert und der Schnitt ihrer Rückseite mit dem Äquatorialkreis der Röhre durch zwei Marken auf der Glaswand bezeichnet. Um die Richtung der Kathodenstrahlen festzulegen, wurde die Röhre in Betrieb gesetzt und die Antikathode soweit gedreht, bis ihre Flächen parallel der Achse des Kathodenstrahlbündels waren, d.h. der Schatten der Schmalseite der Antikathode scharf auf der Wand der Glaskugel in dem Feld der 
von den Kathodenstrahlen bewirkten grünen Glasfluoreszenz erschien. Die Mitte dieses Schlagschattenbildes von $3 \mathrm{~mm}$ Breite wurde auf der Glaswand durch eine Marke festgehalten; dann wurde der Mittelpunkt der Strombasis auf der Kathode, der durch einen dunklen Oxydationsfleck kenntlich war, vertikal von oben her anvisiert und der Schnitt der durch ihn gehenden Vertikalen auf der Wand des Kathodenrohres markiert. Sodann wurde die Kassette in ihre Stellung auf der Holzplatte gebracht und auf diese und das Kathodenrohr ein weißer Karton, welcher eine kreisförmige Öffnung, ein wenig größer als der Äquatorialschnitt der Röntgenröhre, besa $B$, aufgelegt und festgekittet. Mit Hilfe eines kleinen Senkels wurden dann die zuvor auf der Glaswand markierten Punkte übertragen.

Die dergestalt die Richtung der Kathodenstrahlen und die Ebene der Rückseite der Antikathode fixierenden Punkte und der äußere Umfang der Kassette wurden sorgfältig auf einen Zeichenbogen aufgezeichnet und die Punkte miteinander verbunden. Hierbei ergab sich, daß der Schnittpunkt der Achse des Kathodenstrahlbündels und der Ebene der Rückseite der Antikathode zusammenfiel mit dem Mittelpunkt der Glaskugel, und weil die Kassette überall gleichweit entfernt von der Glaskugel aufgestellt war, auch mit dem Mittelpunkt der Kassette und somit der Filmkrümmung. Die Auftreffstelle der Kathodenstrahlen auf der Antikathode, d.h. das Emissionszentrum der Röntgenstrahlen lag demnach um die Dicke der Antikathode, also um 2,6 $\mathrm{mm}$ vor dem Mittelpunkt der Filmkrümmung.

Um die Azimute für die auf den Photogrammen ausgemessenen Schwärzungen zu finden, wurde ein Transporteur so auf das zur Aufsuchung des Emissionszentrums dienende Zeichenblatt gebracht, daß sein Mittelpunkt mit dem Bild des Emissionszentrums der Röntgenstrahlen zusammenfiel, hierauf wurde die Peripherie des einen Halbkreises, für den auf dem Film die Schwärzungen bestimmt worden waren, in Millimeter und der den äußeren Halbumfang der Röntgenröhre andeutende Kreisbogen in $0,5 \mathrm{~cm}$ entsprechend den ausgemessenen Glaswanddicken (vgl. p. 93) eingeteilt.

Es zeigte sich bei der Aufsuchung der Azimutstellung der Platinantikathodenoberfläche auf dem Halbkreis, für den die Schwärzungen bestimmt worden waren, daß dio Platinoberfläche das Azimut $49^{\circ}$ hatte. 
Die Kohleantikathode wurde für eine Reihe von Beobachtungen visuell in dieselbe Stellung wie die Platinantikathode gebracht. Die Vorderseite der Kohleantikathode wies ein Azimut von $48,7^{\circ}$ auf, also um sehr wenig nach kleineren Azimuten zur Stellung der Platinscheibe verschoben, was dadurch bestimmt werden konnte, daß auf je einer Aufnahme von Platinund Kohlestrahlung sich das Schattenbild eines dünnen Platindrahtes befand, der, absichtlich senkrecht zur Äquatorialebene auf die Röhre gekittet und dessen Stellung auf der Glaskugel durch eine Marke festgehalten, mit auf die Zeichnung übernommen worden war. Außerdem wurde bei einer Kohleaufnahme das Azimut $0^{0}$, d.h. die Kathodenstrahlrichtung, durch einen Platinschatten gekennzeichnet. Aus den einzelnen Abständen der Platindrahtschatten und der Grenze der von den Röntgenstrahlen der Vorderfläche herrührenden Schwärzungen auf den Photogrammen konnte mit Hilfe der Zeichnung die Azimutstellung der Kohleantikathode hergeleitet und verglichen werden. In ähnlicher Weise wurde für spätere Beobachtungen, als der Kohleantikathode eine andere Stellung gegeben worden war, das Azimut dieser Stellung ermittelt, und zwar war es $37^{\circ}$.

f) Fehlerquellen geometrischen Ursprungs. Für den Vergleich der Röntgenstrahlschwärzungen und somit indirekt der Röntgenstrahlintensitäten an verschiedenen Stellen des photographischen Films müssen in geometrischer Hinsicht folgende Forderungen erfüllt sein. Erstens muß das Emissionszentrum der Röntgenstrahlen mit dem Zentrum der Filmkrümmung zusammenfallen. Ist dies nicht der Fall, so sind in verschiedenen Azimuten die Entfernung der Filmstellen vom Emissionszentrum verschieden groß und deswegen auch die auf sie fallenden Intensitäten, selbst wenn die Emission vollkommen isotrop ist. Nun war diese Forderung in dem vorliegenden Falle zwar nicht genau, aber doch mit großer Annäherung erfüllt. Die beiden Zentren waren nämlich nur um $2,6 \mathrm{~mm}$ gegeneinander verschoben bei einem Radius der Filmkrümmung von $88 \mathrm{~mm}$. Der dadurch in dem diskutierten Azimutfelde bedingte kleine Fehler war nicht größer als der unvermeidlich kleine Fehler, der daraus sich ergibt, daß das flächenhafte Emissionszentrum nicht genau punktförmig relativ zu seinem Abstand von dem Film ist. Der größte Durchmesser 
der Emissionsfläche betrug nämlich ungefähr $4 \mathrm{~mm}$. Wie leicht zu sehen ist, addieren sich die zwei hier er wähnten kleinen Fehlerbeträge nicht.

Durch das Auseinandertreten von Film- und Emissionszentrum wird noch ein weiterer Fehler bedingt. Ist nämlich die Röntgenröhre in der Äquatorialebene selbst genau kreisförmig, wie es hier wirklich der Fall war, und besitzt sie in dieser Ebene überall dieselbe Wanddicke, so ist der Weg in der Glaswand doch für verschiedene Emissionsazimute verschieden groß, wenn das Emissionszentrum nicht genau mit dem Zentrum des Äquatorialschnittes, also mit dem Zentrum des Films zusammenfällt. Infolge der Verschiedenheit der Wege in der Glaswand ist dann auch die Absorption in dieser und somit die Intensität auf verschiedenen Stellen des Azimuts verschieden groß, selbst wenn die Emission der Röntgenstrahlung vollkommen isotrop ist. In dem Falle der vorliegenden Untersuchung betrug indes selbst die größte Verlängerung des Weges in der Glaswand infolge jener Exzentrizität nur 3 Proz. und war gegenüber der bereits erwähnten Variation der Dicke der Glaswand mit dem Azimut zu vernachlässigen.

Endlich muß dafür gesorgt sein, daß die Röntgenstrahlen in verschiedenen Azimuten die Glaswand genau normal durchlaufen. Denn selbst wenn das Emissionszentrum der Röntgenstrahlen genau mit dem Schnittpunkte verschiedener Beobachtungsrichtungen (Krümmungszentrum des Films) zusammenfällt und selbst wenn mit peinlicher Gewissenhaftigkeit für gleiche Glasdicke in allen untersuchten Emissionsrichtungen gesorgt ist, so werden die Wege in Glas für verschiedene Azimute doch wieder verschieden gro B, wenn die Glaswandstellen nicht alle genau in derselben Neigung durchlaufen werden. Soweit in der vorliegenden Untersuchung in dieser Hinsicht nicht durch das Auseinanderfallen des Emissionszentrums und des Krümmungszentrums der erwähnte kleine Fehler bedingt wurde, kam bei ihr die eben gekennzeichnete Fehlerquelle deswegen nicht in Betracht, weil der Äquatorialschnitt der Röntgenröhre genau kreisförmig und konzentrisch mit dem Film war.

In dieser Hinsicht ist indes, wie gleich hier erwähnt sei, ein dunkler Punkt in der erwähnten Untersuchung von W. Friedrich vorhanden. Dieser Autor hat nämlich an ejner 
käuflichen Röntgenröhre mit Hilfe einer optischen Methode erst die von den Röntgenstrahlen in verschiedenen Azimuten durchlaufenen Wege in der Glaswand bestimmt, dann hat er auf ausgewählte Stellen der Glaswand Glasplättchen von solcher Dicke befestigt, daß für alle Stellen die Summe aus Röntgenstrahlweg in der Glaswand und Dicke des Glasplättchens den gleichen Betrag hatte. Darauf hat er die Glasplättchen auf der Röhrenwand parallel dieser befestigt. Wenn er indes hierbei einen Fehler vermeiden wollte, so mußte or entweder zuvor festgestellt haben, daB die Röntgenstrahlen in verschiedenen Azimuten die Glaswand genau normal durchsetzten, oder wenn dies nicht der Fall war, so durfte er die Kompensationsplättchen nicht parallel der Glaswand, sondern normal zur Emissionsrichtung der Röntgenstrahlen befestigen. Friedrich erwähnt nichts über diesen wichtigen Punkt in seiner Abhandlung. Nun ist eine käufliche Röntgenröhre in der durch die Achse des Kathodenrohres gehenden Ebene in der Regel nicht kreisförmig. Es läßt darum die Arbeit von Friedri ch Zweifel übrig, ob er wirklich die Variation der Wege der Röntgenstrahlen in der Glaswand mit dem Emissionsazimut kompen. siert hat.

g) Gang der Beobachtungen. Während aller maßgebenden Untersuchungen war das Hauptaugenmerk auf das Konstanthalten der jeweiligen Elektrodenspannung an der Röntgenröhre gerichtet. Dies wurde durch Regulierung des Gasdruckes in der Röhre, von dem bekanntlich die Spannung abhängig ist, erreicht. Es wurde nämlich die Röntgenröhre nur so lange unter Strombelastung evakuiert, bis noch merkliche, aber geringe Mengen von Gas aus Antikathode und Glaswand abgegeben wurden, entgegen dem sonstigen Brauch, die Röhre so lange $\mathrm{zu}$ belasten und $\mathrm{zu}$ erwärmen, bis keine bemerkbare Gasabgabe mehr erfolgt. - Dies geschah, um leicht den Gasdruck und damit die Elektrodenspannung regulieren zu können. Während des Stromdurchganges wurde nämlich bei dieser Vorbehandlung der Röhre ebensoviel Gas entwickelt als durch Selbstevakuation verschwand, so daß der Druck von selbst konstant blieb. Und zeigte sich auch nur eine geringe, am Elektrometer beobachtete Abnahme des Kathodenfalles bzw. Zunahme des Druckes in der Röhre, so wurde der zwischen Röhre und Pumpe gelegene Hahn von einem Hilfsarbeiter 
nur einen Augenblick geöffnet, - ein Mittel, das genügte, den Druck und somit den Kathodenfall an der Röhre für die Zeitdauer der eigentlichen Beobachtung in einem Bereiche von 1 Proz. konstant zu halten.

Vor jeder Aufnahme wurde der Abstand der Kugeln in dem Funkenmikrometer soweit vergrößert, daß für die gewänschte Elektrodenspannung an der Röntgenröhre kein Funke im Mikrometer zu befürchten war; außerdem wurde die mit den Spitzen versehene Platte und die dieser gegenüberliegende des Regulators abgenommen, aus dem Grunde, weil diese bei den Vorversuchen infolge starker Ableitung von Elektrizität zu Störungen Anla $B$ gegeben hatten.

Für die Aufnahme wurde sodann der Druck in der Röntgenröhre reguliert, bis die gewünschte Spannung - beobachtet aus dem Ausschlag des Elektrometers - an der Röhre lag. War dies erreicht, so wurde die Influenzmaschine an dem Spitzenregulator kurz geschlossen, die mit Film beschickte im Nebenzimmer bereit gehaltene Kassette um die Glasröhre in der oben geschilderten Weise gelegt und die Exposition begonnen.

Die Belichtungsdauer schwankte je nach der Intensität der Strahlen zwischen einer halben Stunde bis wenigen Sekunden.

Während der Aufnahme wurde andauernd der Ausschlag am Elektrometer beobachtet, und die Elektrodenspannung an der Röntgenröhre, wie schon oben erwähnt, durch kurzes Öffnen des Hahnes zur Quecksilberluftpumpe, welche bei der Aufnahme stets im Betrieb blieb, auf gleicher Höhe gehalten.

Nach Beendigung der Aufnahme wurde das Elektrometer sogleich auf Ladung geprüft - wie dies auch bei langandauernden Belichtungen zwischendurch geschah -, sodann der Ausschlag des Elektrometers mit der Funkenlänge verglichen.

Ich schätze die Genauigkeit der an dem Elektrometer nach jeder photographischen Beobachtung mit Hilfe der Funkenstrecke ermittelten Elektrodenspannung an der Röntgenröhre relativ zu den Werten von Müller ${ }^{1}$ ) auf 3 Proz.

\section{\& 6. Ionisierungsmethode.}

a) Prinzip. Um den „photographischen“ Absorptionsindex der Röntgenstrahlen mit demjenigen vergleichen zu

1) C. Müller, l. c. 
können, welcher sich aus der Messung der von den Röntgenstrahlen bewirkten Gasionisierung ergibt, wurden auch noch Beobachtungen nach dieser Methode angestellt. Es wurde also die gesättigte Stromstärke in einer Gaszelle bestimmt einmal $\left(i_{1}\right)$, wenn ein Al-Blech von bestimmter Dicke in den Strahlengang eingeschaltet war, und einmal $\left(i_{2}\right)$, wenn diese absorbierende Schicht nicht vorhanden war. Die gesättigte Stromstärke ist ja proportional der Ionisierungsstärke, diese wieder proportional der Intensität $(I)$ der Röntgenstrahlung. Es ist somit $I_{1} / I_{2}=i_{1} / i_{2}$, und ist $d$ die durchlaufene Schichtdicke, $k$ der Absorptionsindex, $e$ die Basis der natürlichen Logarithmen, so ist

$$
K=\frac{\log I_{2}-\log I_{1}}{d \log e}=\frac{\log i_{2}-\log i_{1}}{d \log e} .
$$

Da zum Nachweis der Variation des Absorptionsindex mit dem Emissionsazimut die photographische Methode ausreichte, so wurde bei der Ionisierungsmethode darauf verzichtet, den Absorptionsindex für ein schmales Feld von Emissionsazimuten $\mathrm{zu}$ bestimmen. Es wurde lediglich der mittlere

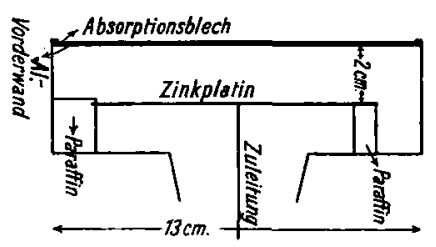

Fig. 6. Absorptionsindex der Röntgenstrahlen in dem Emissionsazimutfeld $56-147^{\circ}$ ermittelt.

b) Ionisierungskammer. Die in der vorliegenden Untersuchung verwendete Ionisierungskammer hatte die Form eines Zylinderkondensators. Sie bestand nämlich aus konzentrischen Kreiszylinderflächen von $91^{\circ}$ Winkelöffnung und $13 \mathrm{~cm}$ Höhe. Die Kammer wurde vertikal und konzentrisch mit der Röntgenröhre aufgestellt, so daB sie das Emissionsazimutfeld von 56-147 ${ }^{\circ}$ füllte. Der Radius der Vorderfläche betrug $18,1 \mathrm{~cm}$, derjenige der Hinterfläche $21,8 \mathrm{~cm}$ (vgl. Fig. 6).

Die Vorderfläche der Kammer bestand aus 0,01 cm dickem Al-Blech, alles übrige aus $0,05 \mathrm{~cm}$ dickem Zinkblech. Am oberen und unteren Rande der Al-Vorderfläche waren Leisten zu dem Zwecke angebracht, Al-Absorptionsbleche von verschiedenen Dicken $(0,01-0,0815 \mathrm{~cm})$, welche seitlich über die Vorderfläche bei den Intensitätsmessungen geschoben 
werden sollten, zu halten und leicht und sicher in den Gang der Strahlen zu bringen.

Parallel und entsprechend zur Al-Vorderfläche gekrümmt, befand sich in $2 \mathrm{~cm}$ Abstand von dieser im Innern des Gehäuses eine $0,1 \mathrm{~cm}$ dicke Zinkplatte, welche vom Boden durch drei $1,8 \mathrm{~cm}$ hohe Paraffinklötzchen isoliert, oben an zwei an der Hinterfläche des Kastens befestigte Paraffinscheibchen angeklebt und von den Seitenwänden und der Decke $1,5 \mathrm{~cm}$ entfernt war.

Als Ionisierungsraum kam demnach der Luftraum zwischen der vorderen Al-Wand und der Zinkplatte in Betracht.

Diese Ionisierungskammer wurde so aufgestellt, daß ihre horizontale Mittellinie in die früher schon genannte Äquatorialebene der Röntgenröhre zu liegen kam, und die Emissionsstelle der Röntgenstrahlen mit dem Mittelpunkte der Krümmung der vorderen Kammerfläche zusammenfiel.

Um eine genau meßbare Stärke des Ionisierungsstromes zu erhalten, wurden die Abmessungen der Ionisierungskammer möglichst groß gewählt. Dabei konnte indes die Höhe nicht größer gewählt werden, ohne daß sich eine Fehlerquelle störend gemacht hätte. Es durchliefen nämlich nu: die Röntgenstrahlen in der Äquatorialebene die vordere Elektrodenfläche, die Luftschicht und das absorbierende Aluminiumblech genau normal. Die übrigen durchliefen sie in schiefer Richtung. Ist also der Winkel eines Strahles mit der Äquatorialebene $\alpha$, $l$ die Dicke des absorbierenden Al-Bleches, $L$ diejenige der Luftschicht in der Ionisierungskammer, so ist der in dieser bzw. in jenem von dem Strahl durchlaufene Weg $L / \cos \alpha$, bzw. $l / \cos \alpha$. Der größte unter den gewählten Maßen vorkommende Winkel $\alpha$ betrug $19^{\circ} 30^{\prime}$, der kleinste Wert von $\cos \alpha$ somit 0,94. War nun der von einem solchen Strahl durchlaufene Weg im Al-Blech gleich $d$ statt $d / 0,94$ so war in Wirklichkeit der damit gemachte Fehler noch kleiner als 6 Proz.; er wurde nämlich zum Teil dadurch kompensiert, daß derselbe Strahl in der Tonisierungskammer einen längeren Weg $L / \cos a$ als ein Äquatorialstrahl zurücklegte und, nachdem er zwar im Verhältnis $1: \cos \alpha$ in Al-Blech stärker absorbiert worden war, in der Luftschicht dafür in demselben Verhältnis mehr ionisierend wirken konnte als ein Strahl in der Äquatorialebene. 
Somit trat die Wirkung der vorstehenden Fehlerquelle in einem zu vernachlässigenden Maße zurück.

c) Schaltung zur Messung der Stärke des Ionisierungsstromes. Das Gehäuse der Kammer war geerdet. Die innere isolierte Zinkplatte war durch eine längere kupferne Leitung mit dem positiven Pol einer Akkamulatorenbatterie von 80 Zellen verbunden. Der ziemlich dicke Zuleitungsdraht zu den Abkumulatoren war durch eine ziemlich weite, $2 \mathrm{~m}$ lange Glasröhre, welche außen vollständig mit geerdetem. Stanniol zum Schutze gegen elektrostatische Störungen umwickelt war, gelegt und in ihrem Innern und Enden von Paraffinscheiben gestützt. Gleich hinter den Akkumulatoren waren durch kurze Kupferdrähte ein Graphitwiderstand von $500000 \mathrm{Ohm}$, dann eine Wippe hintereinander angeschlossen. Von ihrem einen Quecksilbernapf ging eine weitere Zuleitung (1,50 m lang) - in gleicher Weise wie die Zuleitung zu den Akkumulatoren isoliert und von äußeren elektrischen Störungen geschützt zu dem Galvanometer (von Siemens \& Halske), dessen Spule einen Widerstand von $200 \mathrm{Ohm}$ hatte. Zum Zwecke der Dämpfung wurde parallel zur Galvanometerspule ein Widerstand von $10200 \mathrm{Ohm}$ geschaltet.

In dieser Anordnung war die Empfindlichkeit des Galvanometers $8,93 \cdot 10^{-9} \mathrm{Amp}$. für $1 \mathrm{~mm}$ Skalenteil bei $1 \mathrm{~m} \mathrm{Ab}$ stand. Der gewählte Skalenabstand betrug $2,7 \mathrm{~m}$.

Die Akkumulatorenkästen, Graphitwiderstand, Wippe, Galvanometer und Dämpfungswiderstand standen zum Zwecke guter Isolierung von der Erde auf dicken Paraffinplatten. Auf gute metallische Verbindung zwischen den einzelnen Teilen dieser Ausführung war Bedacht genommen; wo eben möglich, waren die Metallteile angelötet oder in Quecksilbernäpfchen getaucht. - Vor den Akkumulatoren usw. war noch eine große geerdete Metallplatte gegen elektrische Störungen aufgerichtet. Der in der vorstehenden Darstellung erwähnte Schutz der Zuleitungen zu den einzelnen Instrumenten bzw. der Akkumulatoren usw. gegen äußere elektrische Einflüsse war vollständig ausreichend. Das Galvanometer blieb nämlich in Ruhe trotz starker Erregung der Influenzmaschine und bei Übergang von Funken in der Funkenstrecke, während der Kasten derselben geöffnet war; ebenso wenn vor das Ioni- 
sierungsgehäuse ein Bleischirm von $3 \mathrm{~mm}$ Dicke gestellt und die Röntgenröhre unter hoher Spannung im Betrieb war.

d) Gang der Beobachtungen. Bei den Messungen des Ionisierungsstromes, des Maßes für die Strahlungsintensität, mußte dafür gesorgt werden, daß außer der Qualität (Absorbierbarkeit) der Röntgenstrahlen auch ihre Intensität konstant blieb. Die erste wird bedingt lurch die Elektrodenspannung, die zweite durch die Stärke des Stromes durch die Röntgenröhre. Es mußte diese deshalb beobachtet werden, besonders schon aus dem Grunde, weil mehrfach eine unregelmäßige Stromlieferung der Influenzmaschine bemerkt worden war. Dies geschah mit Hilfe eines Zeiger-Mikroampèremeters, das hinter der Anode der Röntgenröhre in der Leitung zur Erde lag. Das Amperemeter wurde von dem den Hahn an der Röntgenröhre bedienenden Hilfsarbeiter kontrolliert. Ein zweiter Hilfsarbeiter mußte noch zur Ablesung des Galvanometeraussehlages herangezogen werden. Wie bei den photographischen Beobachtungen, so wurde auch bei dieser elektrischen Methode zur Intensitätsmessung die Spannung an der Röhre durch Regulierung des Druckes (kurzes Óffnen des Hahnes bei betriebener Gaedepumpe) auf der beabsichtigten Höhe gehalten. Hatte die Röhre die gewünschte Spannung, so wurde der Galvanometerausschlag abgelesen und zwar 4-5 mal in kurzen Zwischenpausen, nachdem die Spannung an der Röhre sich geändert hatte und wieder durch Regulierung des Druckes auf denselben Stand gebracht worden war. Aus den so erhaltenen Galvanometerausschlägen wurde das arithmetische Mittel genommen. Oftmals wurde der Nullpunkt des Galvanometers kontrolliert.

\section{Beobachtungen.}

\section{§ 7. Vergleich der Intensitäten der Röntgenstrahlung von Platin und Kohle.}

Wie aus zahlreichen Beobachtungen bereits bekannt ist, nimmt die Intensität der Röntgenstrahlung mit steigendem Atomgewicht der Antikathode zu. Es ist interessant, einmal für eine Reihe von Werten der Elektrodenspannung zwei Antikathoden von sehr verschiedenem Atomgewicht in dieser Hinsicht miteinander zu vergleichen. 
Einen solchen Vergleich ermöglichen die Tab. 2 und Fig. 7. Als Maß der Intensität ist darin die Ionisierungsstromstärke in der benutzten Ionisierungszelle bzw. der ihr proportionale Ausschlag des Galvanometers angegeben.

Tabelle 2.

\begin{tabular}{|c|c|c|c|}
\hline \multicolumn{4}{|c|}{ Röntgenstrahlung } \\
\hline \multicolumn{2}{|c|}{ der Platinantikathode } & \multicolumn{2}{|c|}{ der Kohleantikathode } \\
\hline $\begin{array}{l}\text { Elektroden- } \\
\text { spannung }\end{array}$ & $\begin{array}{l}\text { Galvanometer- } \\
\text { ausschlïge }\end{array}$ & $\begin{array}{l}\text { Elektroden- } \\
\text { spannung }\end{array}$ & $\begin{array}{l}\text { Galvanometer- } \\
\text { ausschläge }\end{array}$ \\
\hline 14380 & 2,1 & 21780 & 1,4 \\
\hline 15080 & 3,6 & 23030 & 1,8 \\
\hline 15840 & 5,8 & 23810 & 3,0 \\
\hline 16540 & 8,5 & 25270 & 3,7 \\
\hline 17300 & 11,0 & 26780 & 4,1 \\
\hline 17740 & 14,0 & 28490 & 4,8 \\
\hline 18750 & 19,4 & 29750 & 6,1 \\
\hline 19750 & 23,3 & 31010 & 6,8 \\
\hline 20500 & 27,2 . & 32320 & 7,3 \\
\hline 21350 & 33,7 & 33480 & 7,8 \\
\hline 22060 & 37,4 & 35650 & 8,5 \\
\hline 23070 & 51,0 & 38160 & 9,6 \\
\hline 24780 & 62,5 & 40480 & 10,2 \\
\hline 26840 & 77,0 & 42890 & 10,8 \\
\hline 27990 & 86,0 & 45260 & 11,1 \\
\hline 29490 & 99,0 & 49780 & 10,7 \\
\hline 30990 & 109,2 & - & 一 \\
\hline 32600 & 115,9 & - & - \\
\hline 33900 & 121,5 & 一 & - \\
\hline 35260 & 130,5 & - & - \\
\hline 37010 & 162,0 & - & - \\
\hline 39320 & 169,3 & - & - . \\
\hline 41980 & 179,5 & - & - \\
\hline 44890 & 182,0 & - & - \\
\hline 47800 & 182,8 & - & - \\
\hline
\end{tabular}

Es braucht wohl kaum bemerkt zu werden, daB die so erhaltenen Intensitäten nicht für verschiedene Werte der Elektrodenspannung miteinander vergleichbar sind, da ja mit dieser sowohl der Absorptionsindex der Glaswand wie derjenige der Luft in der Ionisierungszelle variiert. 
Intensität und Absorptionsindex der Röntgenstrahlen usw. 109

Wohl aber können die Intensitäten der zwei Antikathoden $\left(J_{\mathrm{Pt}}\right.$ bzw. $\left.J_{\mathrm{C}}\right)$ für dieselbe Elektrodenspannung miteinander verglichen werden. Wie aus der Fig. 7 , Kurve $J_{\mathrm{Pt}} / J_{\mathrm{C}}$ hervorgeht, ist das Verhältnis $J_{\mathrm{Pt}} / J_{\mathrm{C}}$ sehr viel größer als 1 und variiert nur wenig mit der Elektrodenspannung. - Die Abweichung bei den niedrigen Spannungen ist wohl durch die

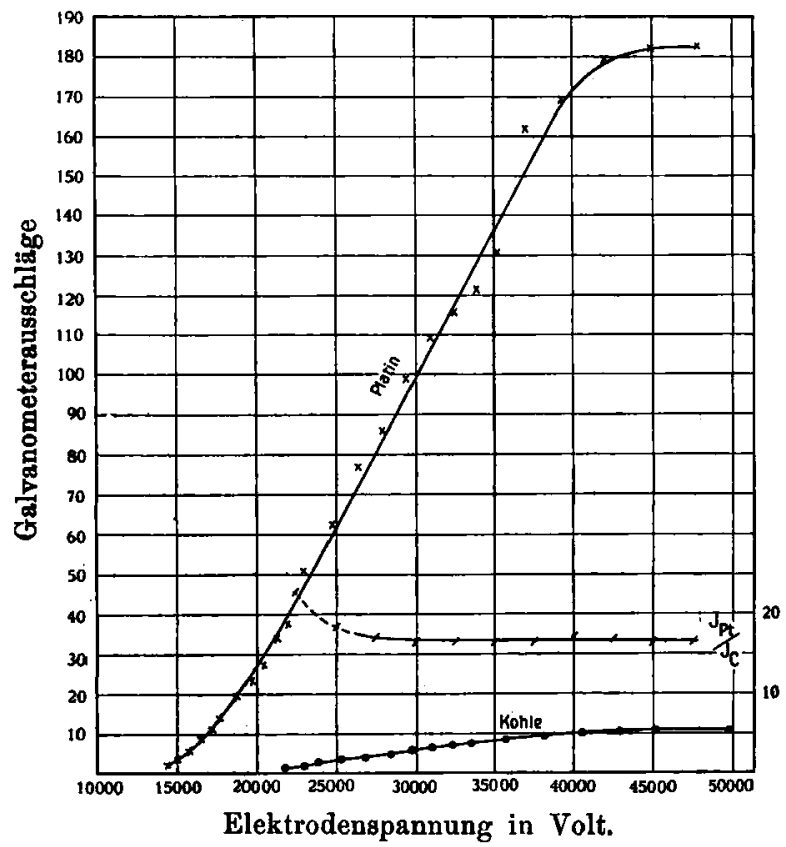

Fig. 7.

Unsicherheit der kleinen Galvanometerausschläge besonders bei Verwendung der Kohleantikathode bedingt.

\section{\$ 8. Der Absorptionsindex der Pt- und C-Btrahlung in Abhängigkeit von der Elektrodenspannung.}

Der Aluminium-Absorptionsindex der Röntgenstrahlung wurde nach der oben angegebenen Ionisierungsmethode sowohl für eine Platin- wie für eine Kohleantikathode bestimmt. Die erhaltenen Werte stellen Mittelwerte dar, sowohl was die Röntgenstrahlungen in verschiedenen Azimuten als auch, was die Strah. 
Tabelle 3.

Pt-Strahlung.

\begin{tabular}{|c|c|c|c|c|}
\hline $\begin{array}{l}\text { Elektroden- } \\
\text { spannung } \\
\text { in Volt }\end{array}$ & $\begin{array}{c}\text { Galvanomete } \\
\text { ohne absorb. } \\
\text { Schirm }\end{array}$ & $\begin{array}{l}\text { rausschläge } \\
\text { mit absorb. } \\
\text { Schirm }\end{array}$ & $\begin{array}{l}\text { Dicke der Al- } \\
\text { Absorptions- } \\
\text { bleche in cm }\end{array}$ & $\begin{array}{c}\text { Absorptions- } \\
\text { index } \\
\mathrm{cm}^{-1}\end{array}$ \\
\hline 14380 & 2,1 & 1,2 & 0,01 & 55,96 \\
\hline 15080 & 3,6 & 2,1 & 0,01 & 53,90 \\
\hline 15840 & 5,8 & 3,5 & 0,01 & 50,50 \\
\hline 16540 & 8,5 & 5,5 & 0,01 & 43,53 \\
\hline 17300 & 11,0 & 7,3 & 0,01 & 41,00 \\
\hline 17740 & 14,0 & 9,4 & 0,01 & 39,84 \\
\hline 18750 & 19,4 & 9,3 & 0,02 & 36,76 \\
\hline 19750 & 23,3 & 12,6 & 0,02 & 30,74 \\
\hline 20500 & 27,2 & 8,4 & 0,04 & 29,38 \\
\hline 21350 & 33,7 & 11,0 & 0,04 & 27,99 \\
\hline 22060 & 37,4 & 13,2 & 0,04 & 26,04 \\
\hline 23070 & 51,0 & 18,8 & 0,04 & 24,95 \\
\hline 24780 & 62,5 & 26,0 & 0,04 & 21,93 \\
\hline 26480 & 77,0 & 18,1 & 0,0815 & 17,77 \\
\hline 27990 & 86,0 & 21,8 & 0,0815 & 16,84 \\
\hline 29490 & 99,0 & 27,4 & 0,0815 & 15,76 \\
\hline 30990 & 109,2 & 33,0 & 0,0815 & 14,86 \\
\hline 32600 & 115,9 & 37,3 & 0,0815 & 13,91 \\
\hline 33900 & 121.5 & 41,5 & 0,0815 & 13,18 \\
\hline 35260 & 130,5 & 45,0 & 0,0815 & 13,06 \\
\hline 37010 & 162,0 & 55,0 & 0,0815 & 13,25 \\
\hline 39320 & 169,3 & 63,0 & 0,0815 & 11,95 \\
\hline 41980 & 179,5 & 72,3 & 0,0815 & 11,16 \\
\hline 44890 & 182,0 & 78,0 & 0,0815 & 10,4 \\
\hline 47800 & 182,8 & 79,8 & 0,0815 & 10,17 \\
\hline
\end{tabular}

lungen verschiedener Absorbierbarkeit in demselben Emissionsazimut betrifft. In Tab. 3,4 und Fig. 8 sind die beobachteten Werte verzeichnet.

Wie aus diesen hervorgeht, ist für alle Elektrodenspannungen 15000 und 50000 Volt der Absorptionsindex der PtStrahlung merklich kleiner als derjenige der C-Strahlung. Bilden wir das Verhältnis der Absorptionsindizes von C- und Pt-Strahlung $\left(K_{\mathrm{C}}\right.$ und $K_{\mathrm{Pt}}$ ) für dieselbe Elektrodenspannung auf Grund der Interpolationskurven, wie es durch die Kurve $K_{\mathrm{C}} / K_{\mathrm{Pt}}$ der Fig. 8 geschehen ist, so zeigt es sich, daß dieses 
Intensität und Absorptionsindex der Röntgenstrahlen usw. 111 Tabelle 4.

C-Strahlung.

\begin{tabular}{|c|c|c|c|c|}
\hline $\begin{array}{l}\text { Elektroden- } \\
\text { spannung } \\
\text { in Volt }\end{array}$ & $\begin{array}{c}\text { Galvanomete } \\
\text { ohne absorb. } \\
\text { Schirm }\end{array}$ & $\begin{array}{c}\text { rausschläge } \\
\text { mit absorb. } \\
\text { Schirm }\end{array}$ & $\begin{array}{l}\text { Dicke der Al- } \\
\text { Absorptions- } \\
\text { bleche in cm }\end{array}$ & $\begin{array}{c}\text { Absorptions- } \\
\text { index } \\
\mathrm{cm}^{-1}\end{array}$ \\
\hline 21780 & 1,4 & 1,0 & 0,01 & 33,65 \\
\hline 23030 & 1,8 & 1,3 & 0,01 & 32,54 \\
\hline 23810 & 3,0 & 2,2 & 0,01 & 31,02 \\
\hline 25270 & 3,7 & 2,8 & 0,01 & 27,87 \\
\hline 26780 & 4,1 & 3,2 & 0,01 & 24,78 \\
\hline 28490 & 4,8 & 3,1 & 0,02 & 21,86 \\
\hline 29750 & 6,1 & 4,1 & 0,02 & 19,87 \\
\hline 31010 & 6,8 & 4,7 & 0,02 & 18,47 \\
\hline 32320 & 7,3 & 5,1 & 0,02 & 17,93 \\
\hline 33480 & 7,8 & 5,6 & 0,02 & 16,57 \\
\hline 35650 & 8,5 & 6,2 & 0,02 & 15,79 \\
\hline 38160 & 9,6 & 7,1 & 0,02 & 15,08 \\
\hline 40480 & 10,2 & 5,6 & 0,04 & 14,99 \\
\hline 42890 & 10.8 & 6,1 & 0,04 & 14,27 \\
\hline 45260 & 11,1 & 6,4 & 0,04 & 13,77 \\
\hline 49780 & 10,7 & 6,3 & 0,04 & 13,24 \\
\hline
\end{tabular}

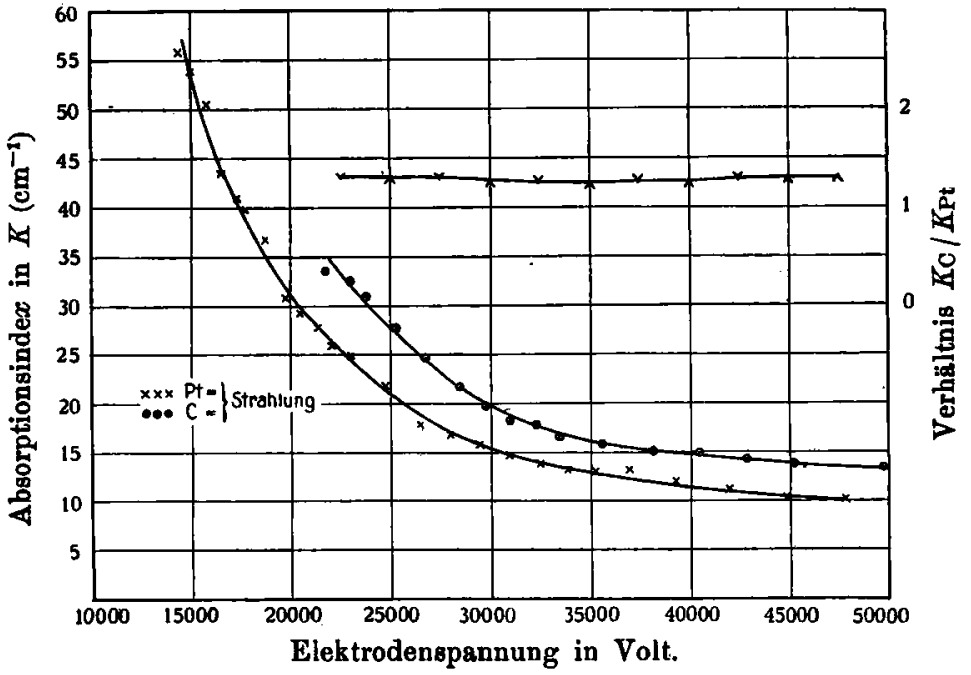

Fig. 8. 
Verhältnis so wenig variiert, daß es für das Gebiet der beobachteten Elektrodenspannungen als konstant angesehen werden kann. Der Wert des Verhältnisses beträgt im Mittel 1,3.

Wie aus der Fig. 8 weiter ersichtlich ist, nimmt sowohl für die Pt- wie für die C-Strahlung der Absorptionsindex $K$ mit wachsender Elektrodenspannung $V$ im Intervall 15000 bis 25000 Volt sehr viel mehr ab als im Intervall 25000-50000 Volt. In dem letzten Intervall ist also die Änderung des Absorptionsindex sehr wenig empfindlich für eine Änderung der Elektrodenspannung.

\section{§ 9. Beziehung zwischen dem elektrischen Absorptionsindex und der speziflschen Schwärzungsdifferenz.}

Der nach der Ionisierungsmethode bestimmte Absorptionsindex der Röntgenstrahlung sei kurz der ,elektrische“ genannt, „photographischer" dagegen derjenige, welcher sich in folgender Weise aus der von der Röntgenstrahlung veranlaßten Entwicklungsschwärzung einer Bromsilbergelatineschicht berechnen läßt.

Bei schwachen Schwärzungen kann wohl die Strahlungs. intensität in erster Annäherung proportional der Schwärzung gesetzt werden, doch ist die Messung schwacher Schwärzungen leicht mit Fehlern behaftet. Darum wurden in der vorliegenden Arbeit nur normale Schwärzungen einer Messung und Diskussion unterzogen. Für sie gilt das von Stark ${ }^{1}$ ) experimentell begründete Schwärzungsgesetz $S=\lg \left(k J^{m} t^{n}\right)$, wo $J$ die Strahlungsintensität, $t$ die Belichtungszeit, $k, m$ und $n$ Konstanten sind. Ist also $J_{1}$ die Intensität hinter einem Al-Blech von der Dicke $d, I_{2}$ die nicht durch dieses geschwächte Intensität, so ist für die gewählte Versuchsanordnung $\left(t_{2}=t_{1}\right)$

oder

$$
\frac{J_{1}}{J_{2}}=e^{-\frac{S_{2}-S_{1}}{m}}=e^{-K d}
$$

$$
K=\frac{S_{2}-S_{1}}{m} \frac{1}{d}
$$

Kennt man darum den Intensitätsexponenten $m$, so läßt sich der photographische Absorptionsindex aus der Schwärzungsdifferenz berechnen. Für die sichtbare Strahlung liegt

1) J. Stark, Ann. d. Phys. 35. p. 461. 1911. 
der Wert von $m$ zwischen 0,5 und 0,7. Von der Bestimmung desselben für die untersuchten Röntgenstrahlungen wurde deswegen abgesehen, weil lediglich ein graphischer Zusammenhang zwischen der Größe $S_{2}-S_{1} / d$ (,,spezifische Schwärzungsdifferenz") und dem elektrischen Absorptionsindex oder eine graphische Eichung der spezifischen Schwärzungsdifferenz auf den elektrischen Absorptionsindex gewonnen werden sollte. Dies wurde in folgender Weise erreicht.

An einer Reihe von Pt- und C-Photogrammen bei verschiedenen Werten der Elektrodenspannung (Tab. 5) wurden

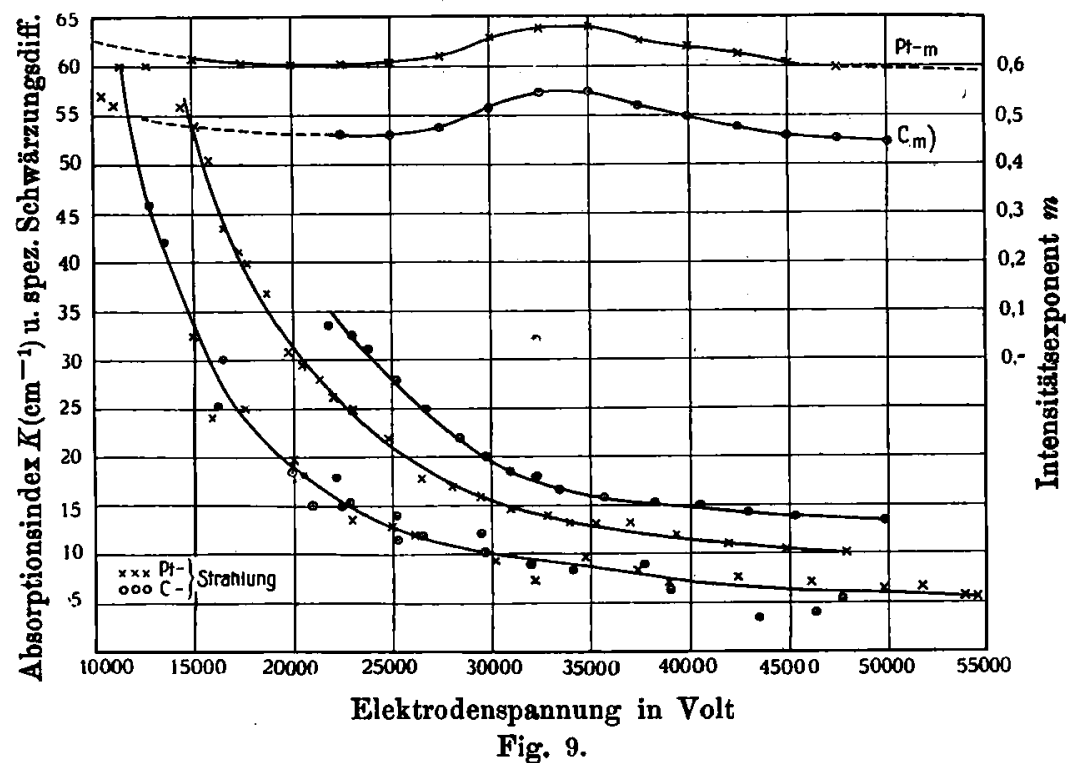

für die senkrecht zum Kathodenstrahlbündel stehende Emissionsrichtung die spezifischen Schwärzungsdifferenzen bestimmt. Diese sind in Fig. 8 eingetragen, ebenso sind in diese die $k$, V-Kurven aus Fig. 9 übernommen und in Fig. 10 ist für die Pt- und C-Strahlung die Beziehung zwischen dem elektrischen Absorptionsindex und der spezifischen Schwärzungsdifferenz auf Grund der Interpolationskurven in Fig. 9 graphisch dargestellt. Hier wird für eine jede Elektrodenspannung einem bestimmten Wert der spezifischen Schwärzungsdifferenz ein bestimmter elektrischer Absorptionsindex 
Tabelle 5 .

Pt-Strablung.

\begin{tabular}{|c|c|c|c|c|}
\hline $\begin{array}{l}\text { Elektroden- } \\
\text { spannung } \\
\text { in Volt }\end{array}$ & $S_{2}$ & $S_{1}$ & $\begin{array}{l}\text { Dicke der Al- } \\
\text { Absorptions- } \\
\text { bleche in cm }\end{array}$ & $\frac{S_{2}-S_{1}}{d}$ \\
\hline 10370 & 1,33 & 0,76 & 0,01 & 57,00 \\
\hline 11060 & 1,52 & 0,96 & 0,01 & 56,00 \\
\hline 11290 & 1,69 & 1,09 & 0,01 & 60,00 \\
\hline 12730 & 2,44 & 1,84 & 0,01 & 60,00 \\
\hline 14980 & 2,8 & 1,5 & 0,04 & 32,5 \\
\hline 15840 & 2,68 & 1,72 & 0,04 & 24,00 \\
\hline 17550 & 2,72 & 1,72 & 0,04 & 25,00 \\
\hline 20040 & 2,68 & 1,89 & 0,04 & 19,75 \\
\hline 23300 & 2,92 & 1,82 & 0,0815 & 13,5 \\
\hline 25020 & 2,48 & 1,44 & 0,0815 & 12,76 \\
\hline 26130 & 2,48 & 1,5 & 0,0815 & 12,02 \\
\hline 30210 & 2,69 & 1,94 & 0,0815 & 9,2 \\
\hline 32240 & 2,17 & 1,58 & 0,0815 & 7,24 \\
\hline 34750 & 2,66 & 1,87 & 0,0815 & 9,69 \\
\hline 37320 & 2,29 & 1,61 & 0,0815 & 8,34 \\
\hline 38930 & 2,24 & 1,66 & 0,0815 & 7,12 \\
\hline 42410 & 2,85 & 2,23 & 0,0815 & 7,61 \\
\hline 46150 & 2,48 & 1,9 & 0,0815 & 7,12 \\
\hline 49800 & 2,09 & 1,58 & 0,0815 & B,26 \\
\hline 51750 & 2,18 & 1,64 & 0,0815 & 6,62 \\
\hline 53950 & 2,16 & 1,7 & 0,0815 & 5,64 \\
\hline 54470 & 2,02 & 1,56 & 0,0815 & 5,64 \\
\hline
\end{tabular}

der Pt- oder der C-Strahlung zugeordnet. Und auf Grund dieser Zuordnung ist es möglich, für eine beobachtete spezifische Schwärzungsdifferenz einen elektrischen Absorptionsindex aufzusuchen. So werden im folgenden die elektrischen Absorptionsindizes in verschiedenen Emissionsazimuten aus den für diese beobachteten spezifischen Schwärzungsdifferenzen ermittelt.

Bevor jedoch dies geschieht, sei auf folgende interessante Beziehungen hingewiesen. Die für die Pt-Strahlung erhaltenen $S_{2}-S_{1} / d, V$-Punkte liegen auf derselben Kurve wie die für die C-Strahlung erhaltenen Werte. Während also die elektrischen Absorptionsindizes der zwei Strahlungen für dieselbe Elektrodenspannung erheblich voneinander verschieden sind, liefern sie dieselbe spezifische Schwärzungsdifferenz. Dieses zunächst 
Intensität und Absorptionsindex der Röntgenstrahlen usw. 115

Tabelle 5 .

C-Strahlung.

\begin{tabular}{c|l|l|c|c}
\hline $\begin{array}{c}\text { Elektroden- } \\
\text { spannung } \\
\text { in Volt }\end{array}$ & $S_{2}$ & $S_{1}$ & $\begin{array}{c}\text { Dicke der Al- } \\
\text { Absorptions- } \\
\text { bleche in cm }\end{array}$ & $\frac{S_{\mathbf{q}}-S_{1}}{d}$ \\
\hline \hline 12860 & 1,79 & 1,33 & 0,01 & 46,00 \\
13650 & 1,58 & 1,16 & 0,01 & 42,00 \\
16200 & 2,03 & 1,02 & 0,04 & 25,25 \\
16450 & 1,62 & 1,32 & 0,01 & 30,00 \\
19930 & 1,89 & 1,16 & 0,04 & 18,5 \\
20890 & 2,03 & 1,43 & 0,04 & 15,00 \\
22170 & 2,34 & 1,62 & 0,04 & 18,00 \\
22400 & 2,02 & 1,42 & 0,04 & 15,00 \\
22810 & 2,08 & 1,46 & 0,04 & 15,5 \\
25260 & 1,96 & 1,4 & 0,04 & 14,00 \\
25300 & 1,79 & 1,33 & 0,04 & 11,5 \\
26570 & 1,87 & 1,39 & 0,04 & 12,00 \\
29540 & 1,91 & 1,43 & 0,04 & 12,00 \\
29670 & 1,61 & 1,21 & 0,04 & 10,00 \\
32010 & 1,9 & 1,19 & 0,0815 & 8,71 \\
34000 & 1,57 & 1,24 & 0,04 & 8,25 \\
37610 & 1,99 & 1,64 & 0,04 & 8,75 \\
38990 & 1,58 & 1,07 & 0,0815 & 6,26 \\
43480 & 1,04 & 0,76 & 0,0815 & 3,44 \\
46320 & 1,03 & 0,7 & 0,0815 & 4,05 \\
47730 & 1,35 & 0,91 & 0,0815 & 5,4 \\
& & & & \\
& & & &
\end{tabular}

überraschende Resultat findet nicht seine Erklärung in der geringeren Empfindlichkeit der photographischen Methode, sondern in folgender Überlegung.

Wegen der selektiven Absorption der Röntgenstrahlung in Elementen von verschiedenem Atomgewicht werden aus einer inhomogenen Strahlung verschiedene Wellenlängen durch zwei verschiedene Elemente im allgemeinen in verschiedenem Verhältnis absorbiert. Der mittlere Absorptionsindex der inhomogenen Strahlung ist also für die zwei Elemente verschieden groß. Werden darum die zwei Elemente zur Intensitätsmessung benutzt, indem die Intensität der einfallenden inhomogenen Strahlung proportional dem absorbierten Betrag gesetzt wird, so ergeben sich für dieselbe primäre Strahlung 
im allgemeinen verschiedene Intensitäten, indem die zur Intensitätsmessung verwandten Elemente auf verschiedene Wellenlängen verschieden ansprechen. In dem vorliegenden Falle dient zur Intensitätsmessung bei der elektrischen Methode die Absorption in den Elementen Stickstoff und Sauerstoff, bei der photographischen Methode die Absorption in den Elementen Brom und Silber im Bromsilberkorn. Nach Barkla ${ }^{1}$ ) weisen diese Elemente abweichend von jenen eine selektive Absorption in dem spektralen Gebiet der Strahlung einer



Fig. ${ }^{\circ} 10$.

Röntgenröhre auf. Aus dem Zusammenfallen der $S_{2}-S_{1} / d$, $V$-Kurven für die Pt- und für die C-Strahlung ist demnach zu folgern, daß das Bromsilber abweichend von der Luft überwiegend nur auf dieselben Wellenlängen der zwei verschiedenen inhomogenen Strahlungen anspricht und darum für diesen denselben mittleren photographischen Absorptionsindex liefert.

Aus vorstehenden Verhältnissen ergibt sich ohne weiteres Iie Folgerung, daß der mittlere photographische Absorptionsindex im allgemeinen verschieden ist von dem mittleren elektrischen Absorptionsindex einer inhomogenen Röntgenstrahlung.

Bemerkenswert ist an der $S_{2}-S_{1} / d, V$-Kurve in Fig.9

1) Ch. G. Barkla, 1. c. 
noch, daß sie im Gebiet 27500-38000 Volt nicht denselben regelmäBigen Verlauf, wie die $K, V$-Kurve aufweist, sondern zwischen zwei allerdings wenig ausgeprägten Wendepunkten etwas flacher als diese verläuft. Dies mag sich daraus erklären, $\mathrm{dab}$ in jenem Gebiet diejenigen Strahlungen, welche $\mathrm{Br}$ oder $\mathrm{Ag}$ oder beide selektiv absorbieren, einen großen Anteil an der Gesamtstrahlung besitzen, so daB infolge der selektiven Absorption die Variation von $K$ kleiner als nach der elektrischen Methode sich ergibt.

Aus der Verbindung der $K, V$ - und $S_{2}-S_{1} / d, V$-Kurven kann endlich der Intensitätsexponent $m$ im Schwärzungsgesetz für den Zweck der Berechnung von Intensitätsverhältnissen gefunden werden. Ist nämlich für einen bestimmten $V$-Wert der elektrische Absorptionsindex $K$, die spezifische Schwärzungsdifferenz $S_{\mathbf{2}}-S_{1} / d$, so ist der Intensitätsexponent $m=S_{2}-S_{1} / d . K$. Dieser Wert ist zwar nur angenähert der wahre Wert des Intensitätsexponenten für die Messung der photographisch wirksamen Intensität, indes erlaubte er eine spezifische Schwärzungsdifferenz in ein Intensitätsverhältnis nach der elektrischen Methode umzurechnen. Für diesen Zweck ist in Fig. 9 die $m, V$-Kurve für die Pt- und C-Strahlung eingetragen.

\section{§ 10. Gesichtspunkte für den Vergleich der Emissionen in verschiedenen Azimuten.}

Auf den ersten Blick mag es aussichtslos oder bedenklich erscheinen, die Emissionen in verschiedenen Azimuten miteinander vergleichen zu wollen, wenn wie bei der in der vorliegenden Arbeit benutzten Röntgenröhre die Dicken der Glaswand nicht für alle Azimute gleich groß sind. Bei genaverem Zusehen ergibt sich indes, daß ein derartiger Vergleich unter den gewählten Versuchsbedingungen gleichwohl im weitgehenden $\mathrm{Maße}$ möglich ist. Es war nämlioh die hierfür wichtige Vorbedingung erfüllt, daB die verwandte Röhre in der untersuchten Äquatorialebene streng kreisförmig war und das Emissionszentrum nahezu mit dem Kugelmittelpunkt zusammenfiel. Außerdem war in dem Azimutfeld um die Emissionsrichtung $90^{\circ}$. senkrecht zur Achse des Kathodenstrahlbündels die Dicke der Glaswand konstant. Dank diesem Umstande kann ohne jede weitere Korrektion festgestellt 
werden, inwieweit die Emission in bezug auf die Ebene senkrecht zur Achse des Kathodenstrahlbündels dissymmetrisch ist. Durch Vergleich von Emissionsazimuten vor und hinter dieser Ebene kann diese Feststellung noch über jenes Azimutfeld konstanter Wanddicke ausgedehnt werden, indem die Emissionen in solchen Azimuten verglichen werden, für welche die Dicken der Glaswand gleich groß sind.

Außer dieser Diskussion, welche sowohl für die Pt- wie für die C-Strahlung an sich vorgenommen werden kann, können noch die Azimut-Verteilungskurven der zwei Strahlungen untereinander verglichen werden.

Was die bei der Diskussion zu berücksichtigenden Fehlerquellen betrifft, so wurden bereits oben die Fehlerquellen geometrischen Ursprungs mit dem Resultat dargelegt, daß ihr EinfluB auf die Resultate der vorliegenden Arbeit vernachlässigt werden kann. Es bleibt noch die Diskussion folgender Fehlerquellen übrig.

Die Glaswand der Röhre sendet infolge der Absorption primärer Röntgenstrahlen nach allen Richtungen sekundäre Strahlen aus. Diese Emission hat indes für ein jedes Emissionsazimut ihr Maximum in diesem selbst, da die dazu geneigten sekundären Strahlen, die aus dem Innern der Glaswand kommen, auf ihrem längeren Wege in dieser eine größere Absorption erfahren. Nun ist die Intensität der sekundären Strahlen proportional derjenigen der primären. Infolge ihres Überwiegens in der Fortpflanzungsrichtung der primären Strahlen und infolge dieser Proportionalität kann darum die Verteilung der Intensität der primären Strahlen nicht in merkbarer Weise durch die sekundären Röntgenstrahlen der Glaswand beeinflußt werden.

Ernster ist der Einfluß der primären Röntgenstrahlen, welche von der Innenseite der Glaswand durch die auf sie treffenden Kathodenstrahlen zur Emission gebracht werden, die von der Antikathode reflektiert werden. Wäre diese Reflexion in der hinter der Ebene senkrecht zur Achse der primären Kathodenstrahlen (Azimut $\varphi<90^{\circ}$ ) größer als vor dieser Ebene (Azimut $\varphi>90^{\circ}$ ), so würde eine in bezug auf diese Ebene dissymmetrische Verteilung der Gesamtstrahlung resultieren, auch wenn die von der Antikathode kommende Röntgenstrahlung für alle Azimute dieselbe Intensität besäße. 
In Wirklichkeit nimmt nach einer Untersuchung von W. Seitz ${ }^{\mathbf{1}}$ ) die Reflexion der Kathodenstrahlen an einer gegen dessen Achse geneigten Platinantikathode von dem Azimut $140^{\circ}$ nach kleineren Azimuten ab, bedingt also eine solche Verteilung der Röntgenstrahlung von seiten der Glaswand, daß für Azimute größer als $90^{\circ}$ die Intensität größer ist als für kleinere Azimute. Wenn wir darum in Wirklichkeit eine Dissymmetrie im umgekehrten Sinne feststellen, so muß diese eine Eigenschaft der von der Antikathode kommenden Röntgenstrahlung sein, sie kann nicht durch die dissymmetrische Reflexion der Kathodenstrahlen vorgetäuscht sein, sie wird vielmehr durch diese verkleinert. In welchem Maße diese Verkleinerung erfolgt, kann leider nicht quantitativ ermittelt werden. Indes ist allein mit der Feststellung einer Dissymmetrie in der Emission von seiten einer Antikathode und dem Vergleich einer Ptund einer C-Antikathode in dieser Hinsicht ein theoretisch wertvolles experimentelles Resultat gewonnen.

\section{\$ 11. Vergleich der Intensitäten der Pt- und C-Strahlung in verschiedenen Fmissionsazimuten.}

In den nachfolgenden Figuren $(11,12,13)$ sind die direkt gemessenen Schwärzungen einer Reihe von Photogrammen längs derselben Schwärzungsskala eingetragen; nur sind die Nullpunkte für die verschiedenen Kurven verschieden gewählt, um diese bequem übereinander anordnen zu können. Um jederzeit auf die gemessenen Schwärzungen $S_{m}$ einer jeden Kurve zurückgreifen zu können, ist einer jeden Kurve die Konstante $k$ beigesetzt, welche die Schwärzungen $(S)$ in der Figur nach der Formel $S_{m}=S \pm k$ in beobachtete Schwärzungen umzurechnen gestattet. - Indes ist die Lage des Nullpunktes der Schwärzungsskala einer jeden Kurve ohne Einfluß auf den Vergleich der Intensitäten verschiedener Azimute einer und derselben Kurve. Dieser Vergleich wird nämlich durch das Verhältnis der Intensität $J_{\varphi}$ eines Azimutes mit einem anderen gegeben.

Gemäß dem oben angegebenen Schwärzungsgesetz ist nämlich

$$
\frac{J_{2}}{J_{1}}=e^{\frac{S_{2}-S_{1}}{m}}
$$

1) W. Seitz, Ann. d. Phys. 6. p. 1. 1901. 
und für Werte von $\left(S_{2}-S_{1}\right) / m$, die beträchtlich kleiner sind als 1 ,

$$
\frac{J_{2}}{J_{1}}=1+\frac{S_{2}-S_{1}}{m}+\frac{1}{2}\left(\frac{S_{z}-S_{1}}{m}\right)^{2} \text {. }
$$

Um eine Änderung des Intensitätsverhältnisses durch eine verschiedene Absorption in der Wand auszuschließen, sind zwei Azimute gleicher Wanddicke miteinander zu vergleichen. Dies ist im folgenden für die Azimute $80^{\circ}$ und $120^{\circ}$ geschehen.



C-Strahlung (Azimut der Antikathode 48,79)

Fig. 11.

Die einer jeden Kurve in den Figg. 11, 12, 13 beigefügte Voltangabe gibt die Elektrodenspannung, für welche sie aufgenommen wurde.

Außerdem ist für eine jede Kurve das Intensitätsverhältnis $J_{\mathbf{8}_{0}} / J_{120}$ angegeben, das mit Hilfe des Intensitätsexponenten aus Fig. 9 nach der vorausgehenden Formel

$$
\frac{J_{80}}{J_{180}}=1+\frac{S_{80}-S_{180}}{m}+\frac{1}{2}\left(\frac{S_{80}-S_{120}}{m}\right)^{2}
$$

berechnet wurde; für kleine und sehr große Elektroden- 
spannungen sind die $m$-Werte extrapolatorisch aus den Kurven entnommen.

Um endlich den Gang der Intensität mit demjenigen der Wanddicke $d$ vergleichen zu können, ist in jede Figur die $d, \varphi$ Kurve $(\odot \odot \odot)$ mit aufgenommen.

Die $S, \varphi$-Kurven der Pt-Strahlung in Fig. 12 setzen sich nicht in das Azimutfeld $0-50^{\circ}$ fort, da in dieses keine Strahlung



Pt-Strahlung.

Fig. 12.

infolge der Absorption in der Pt-Antikathode gelangen kann. Die C-Strahlung dagegen erzeugt nach Fig. 13 auch im Azimutfeld $0-30^{\circ}$ eine Schwärzung, wenn diese hier auch durch die Absorption in der C-Antikathode geschwächt erscheint; das Fehlèn der Schwärzung im Azimutfeld $30^{\circ} \_40^{\circ}$ erklärt sich in diesem Falle aus der Schirmwirkung des die C-Antikathode haltenden Pt-Ringes. Die Neigung der Pt-Antikathode gegen die Richtung der Kathodenstrahlen betrug 49 ${ }^{\circ}$, diejenige der C-Antikathode in Fig. 11 48,7 ${ }^{\circ}$, in Fig. $1337^{\circ}$.

An den $S, \varphi$-Kurven für die Pt-Antikathode sowohl wie 
für die C-Antikathode fällt auf, daß in dem Azimutfeld $70-50^{\circ}$ für $\mathrm{Pt}$ in Fig. 12, in dem Feld $60-40^{\circ}$ für $\mathrm{C}$ in Fig. 13 die Schwärzung mit abnehmendem Azimut rasch kleiner wird, obwohl die Dicke der Glaswand und somit die Absorption in dieser wenig oder gar nicht zunimmt. Diese Erscheinung

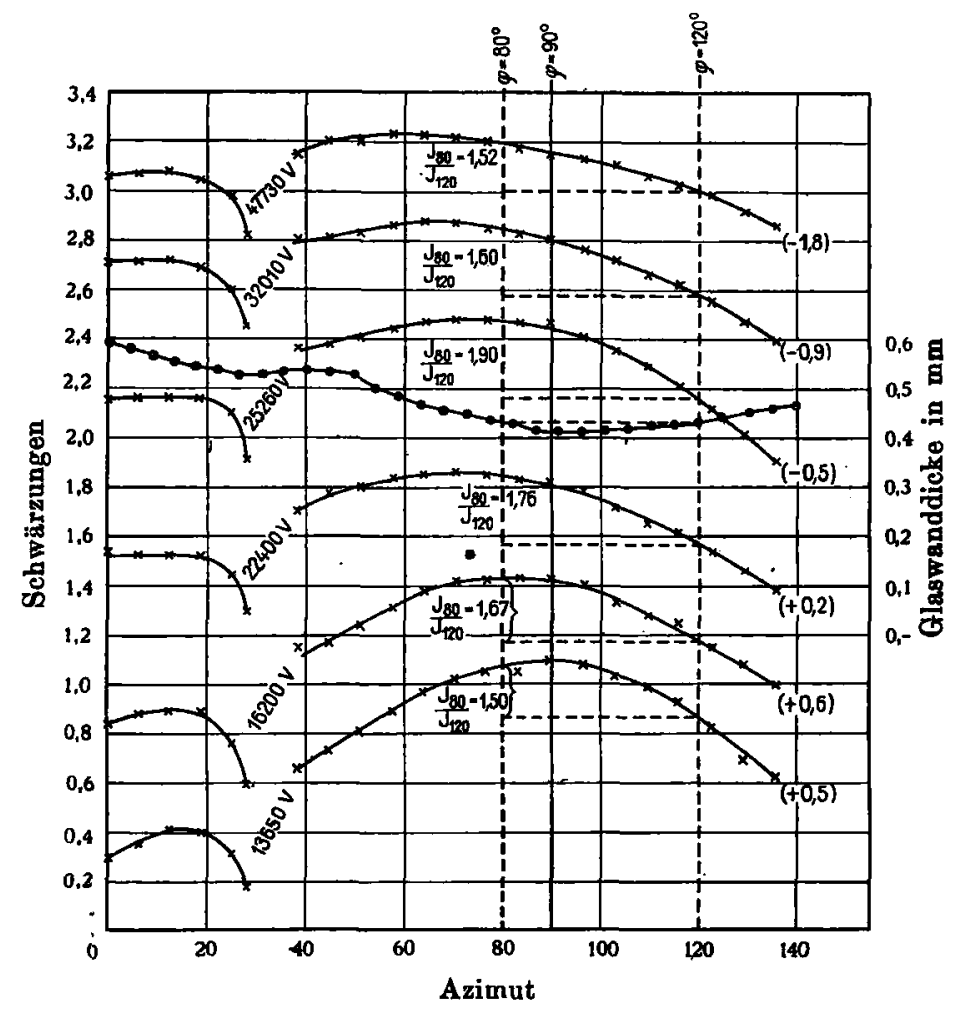

C-Strahlung (Azimut der Antikathode 37\%).

Fig. 13.

erklärt sich offenbar aus der Absorption der schief austretenden Röntgenstrahlung in der Antikathode, wie bereits B. Walter ${ }^{1}$ ) eingehend dargelegt hat.

Wie die Figuren erkennen lassen, ist sowohl für ein Ptwie für eine C-Antikathode die Azimutverteilung der Intensität der Röntgenstrahlung dissymmetrisch in bezug auf die

1) B. Walter, Fortschr. a. d. Geb. d. Röntgenstr. p. 11. 1907. 
Ebene normal zu der Achse der Kathodenstrahlen. Es ist nämlich das Verhältnis $J_{80} / J_{120}$ für die Pt- wie für die C-Strahlung größer als 1; für die C-Strahlung liegt sein Wert zwischen 1,50 und 1,90, für die Pt-Strahlung zwischen 1,07 und 1,28. Für beide Strahlungen scheint er mit wachsender Elektrodenspannung erst bis zu einem Maximum zu- und dann wieder etwas abzunehmen. Die grobe Dissymmetrie für die C-Strahlung steht in Übereinstimmung mit den Resultaten Starks ${ }^{1}$ ) für eine C-Antikathode. Nach Friedrich ${ }^{\mathbf{1}}$ ) beträgt das Verhältnis $J_{80} / J_{120}$ für eine Pt-Antikathode 1,08; dieser Wert stimmt nur mit dem für eine hohe Elektrodenspannung hier gefundenen Wert 1,07 gut überein. Friedrich hat die in seinen Beobachtungen gewählte Elektrodenspannung nicht genau gemessen, sondern nur mit einem Funkenmikrometer angenähert bestimmt. Da er für jene Werte des Verhältnisses $J_{80} / J_{120}=1,08$ eine Elektrodenspannung von 50000 Volt angibt, so ist die UUbereinstimmung zwischen dem hier für 53950 Volt gefundenen Wert 1,07 eine ziemlich gute. Für erheblich kleinere Werte der Elektrodenspannung hat Friedrich keine Beobachtungen mitgeteilt.

In Fig. 11 sind mehrere Beobachtungsreihen für eine C-Antikathode mitgeteilt, deren Ebene ein Azimut von 48,7 besa $B$, bei welcher sich also die Absorption der Röntgenstrahlung in ihr selbst schon für kleinere Azimute bemerkbar machte. Wie ein Vergleich mit Fig. 13 lehrt, ist eine gute Übereinstimmung in den Werten von $J_{\mathbf{8} 0} / J_{120}$ vorhanden. Nur sind diese infolge der Absorption in der Antikathode im ersten Falle durchweg etwas kleiner als im zweiten.

\section{\$ 12. Vergleich der Absorptionsindizes in versohiedenen Emissionsazimuten.}

Um die Absorptionsindizes in verschiedenen Azimuten miteinander vergleichen zu können, sind in der oben angegebenen Weise mit Hilfe der Fig. 10 die spezifischen Schwärzungsdifferenzen in elektrische Absorptionsindizes umgerechnet und die erhaltenen Werte in den Figg. 14, 15 graphisch dargestellt worden. Es ist aus diesen zunächst ersichtlich, daß der Absorptionsindex, wie es $\mathrm{zu}$ erwarten stand, empfindlich mit der Dicke der Glaswand variiert und zwar um so kleiner

1) J. Stark, l. c.; W. Friedrioh, l.c. 
erscheint, je größer die durchlaufene, absorbierende schichtdicke ist, eine Erscheinung, welche bereits von Röntgen aufgefunden und in der bekannten Weise aus der Inhomogenität der Strahlung einer Antikathode erklärt worden ist.

Vergleichen wir die Absorptionsindizes für die Azimute $80^{\circ}$ und $120^{\circ}$ gleicher Glaswanddicke, so ergibt sich zwar für die kleinsten Elektrodenspannungen (22600 bzw. 25020 Volt) im Falle der C- und der Pt-Strahlung für $80^{\circ}$ ein kleinerer Absorptionsindex als für $120^{\circ}$. Indes ist der Unterschied nur

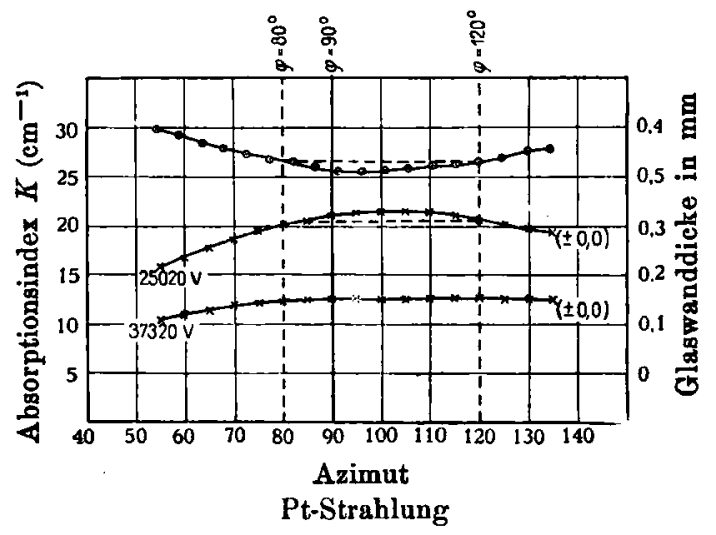

Fig. 14.

klein und für größere Elektrodenspannung ist ein Unterschied überhaupt nicht mehr mit Sicherheit festzustellen.

Stark hat einen viel größeren Unterschied der Absorptionsindizes auf photographischem Wege gefunden. Indes hat er mit kleineren Schwärzungen als ich gearbeitet, demgemäB nicht mit dem Gesetz für die Normalschwärzungen gerechnet und darum die Schwärzung angenähert proportional der Intensität gesetzt. Da Hrn. Prof. Stark dieses Verfahren leicht zu Fehlern Anlaß geben kann, so habe ich auf seinen Vorschlag das in der vorliegenden Untersuchung ausgearbeitete Verfahren angewandt. Im Einverständnis mit Hrn. Prof. Stark teile ich mit, daß nach seiner Ansicht sein früher erhaltenes Resultat über die Abhängigkeit des Absorptionsindexes von dem Azimut durch das obige Resultat zu ersetzen ist.

Friedrich hat über die Absorption der Röntgenstrahlen in verschiedenen Emissionsazimuten durch eine $0,666 \mathrm{~mm}$ 
dicke Glasplatte und eine $0,0025 \mathrm{~mm}$ dicke Platinfolie $\mathrm{Be}$ obachtungen mitgeteilt. Nun sind seine Angaben für das - Azimut $55^{\circ}$ zweifellos bereits durch die Absorption in der Pt-Antikathode beeinflußt; wahrscheinlich auch schon, obgleich viel weniger, die Angaben für $70^{\circ}$. Berechnet man durch Interpolation aus seinen Angaben für $133^{\circ}, 103^{\circ}$ und $70^{\circ}$ die prozentische Absorption für $120^{\circ}$ und $80^{\circ}$, so ergeben sich nach seinen zwei Tabellen die Werte 34,7 und 31,7 Proz.

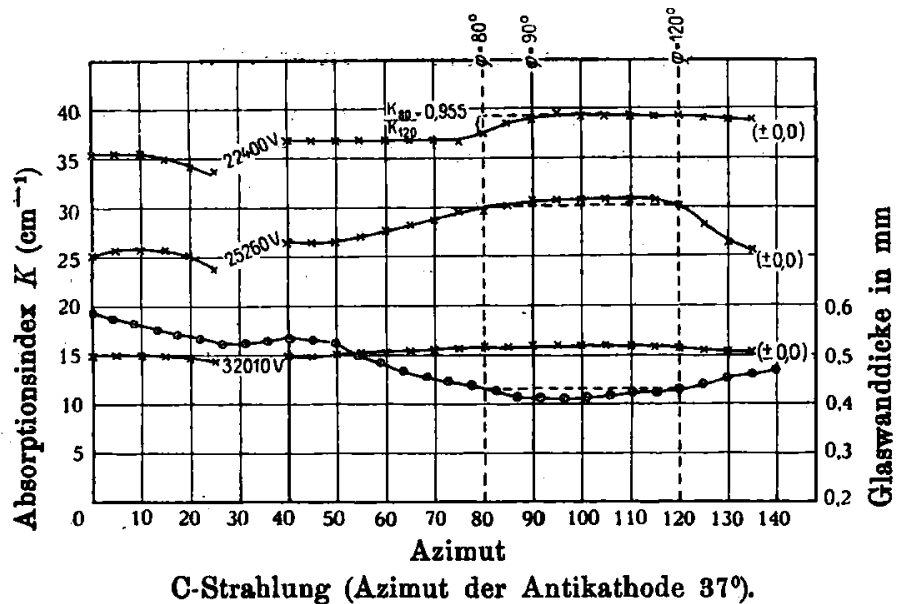

Fig. 15.

bzw. 28,85 und 27,0 Proz. Die sich hieraus ergebende Verschiedenheit der Absorptionsindizes ist zwar ebenfalls klein, aber doch noch merklich. Und zwar betrug hierbei die Elektrodenspannung 45000-46000 Volt. Für 32000 Volt habe ich indes eine Verschiedenheit der Absorptionsindizes in den zwei Azimuten nicht mehr mit Sicherheit feststellen können. Vielleicht erklärt sich diese kleine Differenz in den beiderseitigen Angaben daraus, daß für das zur Interpolation benutzte Azimut die Absorption der Röntgenstrahlung in der Pt-Antikathode die Strahlung bereits härter gemacht hat.

Wenn somit nur für eine Elektrodenspannung kleiner als 25000 Volt durch meine Beobachtungen sicher erwiesen ist, daß der Absorptionsindex für Azimute unter $90^{\circ}$ ein wenig kleiner ist als für Azimute über $90^{\circ}$, so darf daraus nicht allein für Elektrodenspannungen unter 25000 Volt gefolgert werden, daß die Frequenz der wirksamen Strahlung für Azimute unter 
$90^{\circ}$ größer ist als für Azimute über $90^{\circ}$. Das wird zweifellos auch für Elektrodenspannungen über 25000 Volt der Fall sein; nur ist hier die Messung des Absorptionsindex eine ungenaue Methode für den Nachweis des Unterschiedes der Frequenzen. Denn wie sich oben ergab, ändert sich oberhalb 25000 Volt der Absorptionsindex nur wenig mit der Elektrodenspannung und somit wahrscheinlich auch nur wenig mit der Frequenz der Röntgenstrahlung.

\section{§ 13. Azimut größter Intensität.}

Würde der Absorptionsindex der von der Antikathode kommenden anisotropen Strahlung beträchtlich mit dem Azimut variieren, so würde das Azimut des Intensitätsmaximums durch die Absorption in einer überall gleich dicken Glaswand merklich vergrößert werden. So aber ist nach dem vorstehenden Resultat der Absorptionsindex nur sehr wenig für benachbarte Azimute verschieden. Darum kann die Lage des Intensitätsmaximums nicht merklich von der Anisotropie des Absorptionsindex beeinflußt werden. Aus diesem Grunde läßt sich über die Lage des Intensitätsmaximums für eine Kohleantikathode nachstehende quantitative Aussage machen.

Bei der von mir benutzten Röntgenröhre nimmt in dem Azimutfeld $80-50^{\circ}$ die Dicke der Glaswand und somit die Absorption in dieser und ebenso die Absorption in der Antikathode in jenem Felde mit abnehmendem Azimut zu. Infolge dieser zunehmenden Absorption nimmt die Intensität der außerhalb der Röhre beobachteten Strahlung mit abnehmendem Azimut ab, obwohl sie ohne das Dazwischentreten jener zwei Absorptionen jedenfalls bis zu einem Maximum zunehmen würde. Ist also in dem Azimutfeld 80-50 ein Intensitätsmaximnm wirklich vorhanden, so muß das beobachtete ,scheinbare" Intensitätsmaximum immer bei einem erheblich größeren Azimut auftreten als jenes "wirkliche“" Intensitätsmaximum der durch die Absorption in ihrer Verteilung nicht gestörten Strahlung.

In Tab. 6 und Fig. 16 ist nun für die zwei Azimute (37 ${ }^{\circ}$ und $48,7^{\circ}$ ) der C-Antikathode das Azimut des scheinbaren Intensitäts- oder Schwärzungsmaximums als Funktion der Elektrodenspannung dargestellt. Wie man sieht, ist für beide Azimute der Antikathode die Abnahme des Azimuts 
Intensität und Absorptionsindex der Röntgenstrahlen usw. 127

des Maximums zunächst proportional der Zunahme der Elektrodenspannung, von etwa 25000 Volt ab ist indes die Abnahme

Tabelle 6.

\begin{tabular}{|c|c|c|c|}
\hline \multicolumn{2}{|c|}{$\begin{array}{l}\text { C-Antikathode } \\
\text { mit Azimutstellung } 48,7^{\circ}\end{array}$} & \multicolumn{2}{|c|}{$\begin{array}{l}\text { C-Antikathode } \\
\text { mit Azimutstellung } \mathbf{3 7}^{\circ}\end{array}$} \\
\hline $\begin{array}{l}\text { Elektroden- } \\
\text { spannung in Volt }\end{array}$ & $\begin{array}{l}\text { Azimut des } \\
\text { Intensităts- } \\
\text { maximums }\end{array}$ & $\begin{array}{l}\text { Elektroden- } \\
\text { spannung in Volt }\end{array}$ & $\begin{array}{l}\text { Azimut des } \\
\text { Intensitäts- } \\
\text { maximums }\end{array}$ \\
\hline 12860 & 90,0 & 13650 & 89,0 \\
\hline 16450 & 87,0 & 16200 & 82,0 \\
\hline 20890 & 77,5 & 19930 & 82,0 \\
\hline 25300 & 73,0 & 22170 & 70,5 \\
\hline 29670 & 69,0 & 22400 & 70,0 \\
\hline 34000 & 66,0 & 22810 & 70,0 \\
\hline 37610 & 65,0 & 25260 & 70,0 \\
\hline 38990 & 69,0 & 26570 & 69,5 \\
\hline 43480 & 66,5 & 29540 & 67,5 \\
\hline 46320 & 72,5 & 32010 & 64,5 \\
\hline- & - & 47730 & 58,0 \\
\hline
\end{tabular}

langsamer, erreicht für das größere Antikathodenazimut den Wert Null bei etwa $\mathbf{3 6 0 0 0}$ Volt und von da ab wird das Azimut des Maximums sogar wieder größer. Dieses letzte singuläre

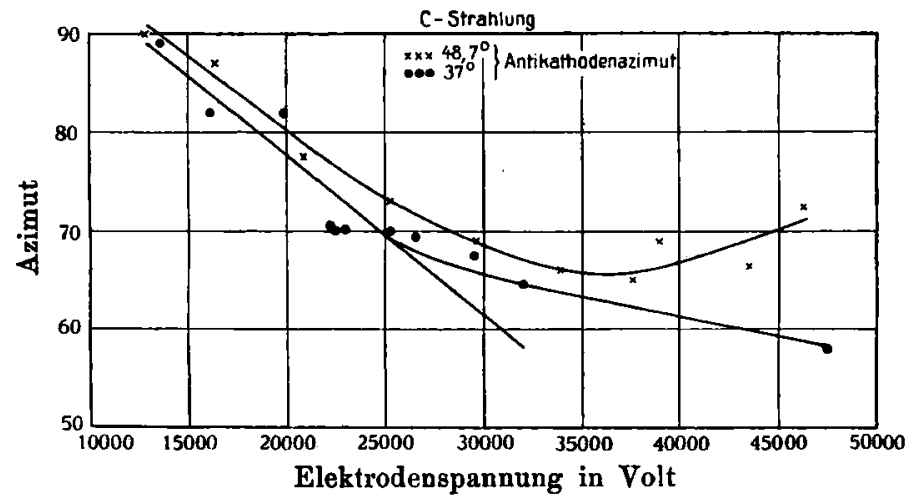

Fig. 16.

Verhalten sowie der Umstand, daß die Azimute maximaler Intensität für das größere Antikathodenazimut größer sind als diejenigen für das kleinere Antikathodenazimut, erklären 
sich offenbar aus der Röntgenstrahlenabsorption in der Antikathode. Dies ist für das größere Antikathodenazimut größer als für das kleinere. Sie nimmt ferner $\mathrm{zu}$, je tiefer die $\mathrm{Ka}$ thodenstrahlen mit wachsender Geschwindigkeit, also mit wachsender Elektrodenspannung in die Antikathode eindringen. Die Zunahme dieser Absorption aber bedeutet Zurückdrängen des Intensitätsmaximums nach größeren Azimuten.

Für eine quantitative Diskussion müssen die Punkte der Kurve oberhalb der Elektrodenspannung 30000 Volt ausscheiden. Und auch die Azimute für kleinere Werte der Elektrodenspannung sind nur obere Grenzwerte für die wirklichen Azimute maximaler Intensität. Eine lineare Extrapolation von dem Intervall $18000-25000$ Volt nach 28000 Volt zur Elimination der Antikathodenabsorption liefert als scheinbares Azimut für diese Spannung den Wert 64,7 ${ }^{\circ}$. Das wirkliche Azimut maximaler Intensität für 28000 Volt ist also kleiner als $64,7^{\circ}$. Nun ist nach der von A. Sommerfeld ${ }^{1}$ ) entwickelten Impulstheorie der Röntgenstrahlen (nach Fig. 4 der Abhandlung von Sommerfeld) das Azimut maximaler Intensität $66^{\circ}$ für eine Kathodenstrahlgeschwindigkeit von $1 \cdot 10^{10} \mathrm{~cm} \mathrm{sec}^{-1}$ oder einen Kathodenfall von 28000 Volt, also gröBer als der hier experimentell gefundene obere Grenzwert dieses Azimuts.

Dieses Resultat steht in Übereinstimmung mit demjenigen, welches $\mathrm{Stark}^{2}$ ) für eine Antikathode aus Holzkohle erhielt. Nur fand Stark noch etwas kleinere Werte für das Azimut maximaler Intensität. Dies mag sich daraus erklären, daß die Röntgenstrahlenabsorption in der von ihm verwendeten Holzkohle beträchtlich kleiner war als in der hier benutzten Retortenkohle.

\section{Resultate.}

1. Bei gleicher Elektrodenspannung ist die Pt-AntikathodenRöntgenstrahlung ungefähr $17 \mathrm{mal}$ intensiver als die einer C-Antikathode.

2. Sowohl die Pt- wie die C-Strahlung wird dissymmetrisch in bezug auf eine Ebene senkrecht zur Achse der erzeu-

1) A. Sommerfeld, Phys. Ztschr. 10. p. 969.1909.

?) J. Stark, Phys. Ztschr. 10. p. 902. 1909; 11. p. 107. 1910. 
genden Kathodenstrahlen emittiert. Das Intensitätsverhältnis $J_{80} / J_{120}$ liegt bei $12000-50000$ Volt Elektrodenspannung für eine C-Antikathode zwischen 1,50 und 1,90, für eine Platinantikathode zwischen 1,07 und 1,28.

3. Der elektrische Absorptionsindex der Röntgenstrahlung ist im allgemeinen verschieden von dem photographischen Absorptionsindex.

4. Der Al-Absorptionsindex der C-Strahlung ist für alle untersuchten Elektrodenspannungen größer als derjenige der Pt-Strahlung etwa im Verhältnis $1: 0,77$.

5. Im Intervall 12000-25000 Volt Elektrodenspannung ist der Absorptionsindex empfindlich mit dieser variabel, mit wachsender Elektrodenspannung wird er indes nur sehr langsam kleiner.

6. Für Elektrodenspannungen kleiner als 25000 Volt ist der Absorptionsindex etwas, aber nur wenig mit dem Azimut veränderlich. $k_{80} / k_{120}$ beträgt für die C-Strahlung 0,955.

7. Für eine Elektrodenspannung von 28000 Volt ist das Azimut maximaler Intensität kleiner als $66^{\circ}$.

Die vorstehende Arbeit wurde im physikalischen Institut der Königl. Technischen Hochschule in Aachen vom Herbst 1911 bis Sommersemester 1913 auf Anregung und unter Leitung von Hrn. Professor Dr. Stark ausgeführt.

(Eingegangen 26. September 1914.) 